\title{
Theoretical Understanding of the Linear Relationship between Convective Updrafts and Cloud-Base Height for Shallow Cumulus Clouds. Part I: Maritime Conditions
}

\author{
YOUTONG ZHENG \\ Earth System Science Interdisciplinary Center, University of Maryland, College Park, College Park, Maryland
}

(Manuscript received 2 November 2018, in final form 22 May 2019)

\begin{abstract}
Zheng and Rosenfeld found linear relationships between the convective updrafts and cloud-base height $z_{b}$ using ground-based observations over both land and ocean. The empirical relationships allow for a novel satellite remote sensing technique of inferring the cloud-base updrafts and cloud condensation nuclei concentration, both of which are important for understanding aerosol-cloud-climate interactions but have been notoriously difficult to retrieve from space. In Part I of a two-part study, a theoretical framework is established for understanding this empirical relationship over the ocean. Part II deals with continental cumulus clouds. Using the bulk concept of mixed-layer (ML) model for shallow cumulus, I found that this relationship arises from the conservation law of energetics that requires the radiative flux divergence of an ML to balance surface buoyancy flux. Given a certain ML radiative cooling rate per unit mass $Q$, a deeper ML (higher $z_{b}$ ) undergoes more radiative cooling and requires stronger surface buoyancy flux to balance it, leading to stronger updrafts. The rate with which the updrafts vary with $z_{b}$ is modulated by $Q$. The cooling rate $Q$ manifests strong resilience to external large-scale forcing that spans a wide range of climatology, allowing the slope of the updrafts $-z_{b}$ relationship to remain nearly invariant. This causes the relationship to manifest linearity. The physical mechanism underlying the resilience of $Q$ to large-scale forcing, such as free-tropospheric moisture and sea surface temperature, is investigated through the lens of the radiative transfer theory (two-stream Schwarzschild equations) and an ML model for shallow cumulus.
\end{abstract}

\section{Introduction}

Shallow cumulus clouds play an important role in Earth's radiation budget (Hartmann et al. 1992; Rossow and Schiffer 1999). They are sustained by convective updrafts that feed moisture and cloud condensation nuclei $(\mathrm{CCN})$ to the clouds. Convective updraft speed has received attention not commensurate with its critical significance in understanding the aerosol-cloud interactions and their climatic effects (Rosenfeld 2014; Rosenfeld et al. 2014b; Donner et al. 2016; Seinfeld et al. 2016). A key reason for the underexploration of convective updrafts is the notorious difficulty of measuring them. Without sufficient observations, numerical modeling of updrafts remains poorly constrained (Donner et al. 2016). It is even suggested that the low fidelity of atmospheric models in simulating the updrafts gives rise to the large uncertainties with which the climate forcing and sensitivity are estimated (Donner et al. 2016).

Corresponding author: Youtong Zheng, zhengyoutong@ gmail.com
Recent progresses in satellite-based inference of convective updrafts (Zheng and Rosenfeld 2015; Zheng et al. 2015, 2016) attempt to remedy, if not fill, this observational gap. The key idea of these techniques is that, instead of directly measuring the updrafts that proves to be expensive and limited in temporal and spatial coverage, we can infer them by quantifying the convective energy that propels the updrafts. The convective energy is estimated from physical variables that are easier to obtain such as surface temperatures (Zheng et al. 2015), cloud-base height (Zheng and Rosenfeld 2015), and cloud-top radiative cooling for stratocumulus (Zheng et al. 2016). These energy-quantification methods inevitably rely, to a varying degree, on empirically determined parameters and relationships. Among these relationships, the most intriguing and perhaps promising one is the linear relationship between the updrafts and cloud-base heights (Zheng and Rosenfeld 2015) because of its simplicity and validity over both land and ocean. Moreover, with emerging satellite imagers with highresolution infrared channels [e.g., Visible Infrared Imaging Radiometer Suite (VIIRS) onboard the Suomi 
NPP satellite], it has become possible to retrieve the convective cloud-base temperature and height $z_{b}$ with high accuracy (Zhu et al. 2014). With the $z_{b}$, the updrafts$z_{b}$ linear relationship allows the updrafts to be inferred (Zheng and Rosenfeld 2015). This concept of updrafts remote sensing has been applied to a new method of satellite retrieval of $\mathrm{CCN}$ concentration by using natural clouds as CCN chambers (Rennó et al. 2013; Rosenfeld et al. 2014a, 2016).

This updrafts $-z_{b}$ relationship has been applied universally regardless of the underlying surface type: land or ocean (e.g., Rosenfeld et al. 2016, 2019; Yue et al. 2019). Experimental data show that the relationship of cloudbase updrafts versus $z_{b}$ is $\sim 20 \%$ steeper over land than that over the ocean, but the scatter of the samples, in particular over land, is too large for such a difference to achieve statistical significance (Zheng and Rosenfeld 2015). Is the statistical indifference physical? Or are there unnoticed influential factors that regulate updrafts without affecting $z_{b}$, or vice versa, causing a more scattered relationship over land? In either case, a well-established theoretical framework that elucidates the physical mechanisms behind the relations are still lacking, which motivates the current study. This paper, as Part I of a two-part study, focuses on shallow cumulus over the ocean. Part II deals with continental clouds. It makes physical sense to distinguish between cumulus clouds in maritime and continental conditions because the ocean has a much larger thermal inertia. Marine boundary layers are typically considered as quasi-steady systems whereas continental boundary layers are more unsteady and more strongly forced by the surface sensible heat fluxes that change rapidly at a diurnal scale. Marine clouds might be an appropriate starting point for studying this problem because equilibrium assumption markedly simplifies the analytic treatment of the system. Moreover, this study uses the Deardorff convective velocity scale $w^{*}$ (Deardorff 1970) to approximate the strength of updrafts; $w^{*}$ has been shown to be a good approximation of convective updrafts (Fig. 4a in van Stratum et al. 2014), but only when the surface fluxes are stationary (van Driel and Jonker 2010). Under nonstationary surface forcing, such as in decay phase of a continental cumulus diurnal cycle, $w^{*}$ becomes less valid (Nieuwstadt and Brost 1986; Sorbjan 1997). Therefore, as a first step, I take advantage of the near-equilibrium nature of marine boundary layers so as to establish a heuristic conceptual framework, based on which the more complicated conditions over land will be investigated in Part II.

Here, three scientific questions are of particular interest:

1) Why is there a positive correlation between the updrafts and cloud-base heights over the ocean?

2) Why does the relationship appear linear?
3) How sensitive is the relationship to external largescale forcing?

While the first question has been touched upon in previous studies including our own (Golaz et al. 2001; Williams and Stanfill 2002; Stevens 2007; Zheng and Rosenfeld 2015), the other two remain unexploited. An existing theoretical idea from Williams and Stanfill (2002, hereafter WS02) stresses the role of Bowen ratio that controls both parameters. Specifically, over a dry surface with large Bowen ratio, the lifting condensation level, as a good proxy for $z_{b}$, is higher. Under the same degree of solar insolation, higher Bowen ratio corresponds to stronger surface sensible heat fluxes that drive more strongly buoyant boundary layer parcels. As will be shown later, this mechanism can be important over land surface, but not so over ocean.

Figure 1 illustrates the schematic diagram of a conceptual model (Neggers et al. 2006) used in this study for addressing these questions. This conceptual model is an inheritance from traditional bulk mixed-layer (ML) models (Lilly 1968; Betts 1973; Stevens 2006; Tennekes and Driedonks 1981). The ML (or subcloud layer) is considered as a bulk of thermodynamically nonstratified fluid that is warmed by surface fluxes and entrainment and cooled by the longwave radiation. A unique aspect of this ML model is that it allows mutual interactions between the ML and cumulus clouds that cap the ML: cumulus mass fluxes can shallow the ML while the energetics and moisture content of the ML modulate the cumulus mass fluxes and cloud fractional coverage, respectively. An important consequence of the interactions is that the base of the cumulus always finds itself adjacent to the top of the ML in an equilibrium state [see Neggers et al. (2006) for details]. The proxy between $z_{b}$ and ML depth $h$ allows $z_{b}$ to be linked with the other properties of the ML (e.g., energetics), as will be shown in the next section.

In the next section, I will derive an analytical relationship between the $w^{*}$ and $z_{b}$. Based on the derived simple formula, we can gain a preliminary understanding of the first two scientific questions. Then I examine the ML radiative cooling rate, a parameter that is central for understanding this relationship, by analytically exploring its dependence on internal parameters of the ML system and on external forcing. Finally, I couple the formula of radiative cooling with the shallow-cumulus-topped ML model (Neggers et al. 2006) to address the second and third scientific questions. Finally, the "Bowen ratio argument" from WS02 is revisited, followed by conclusions in the end.

\section{The theoretical framework}

The Deardorff convective velocity scale (Deardorff 1970) is defined as 


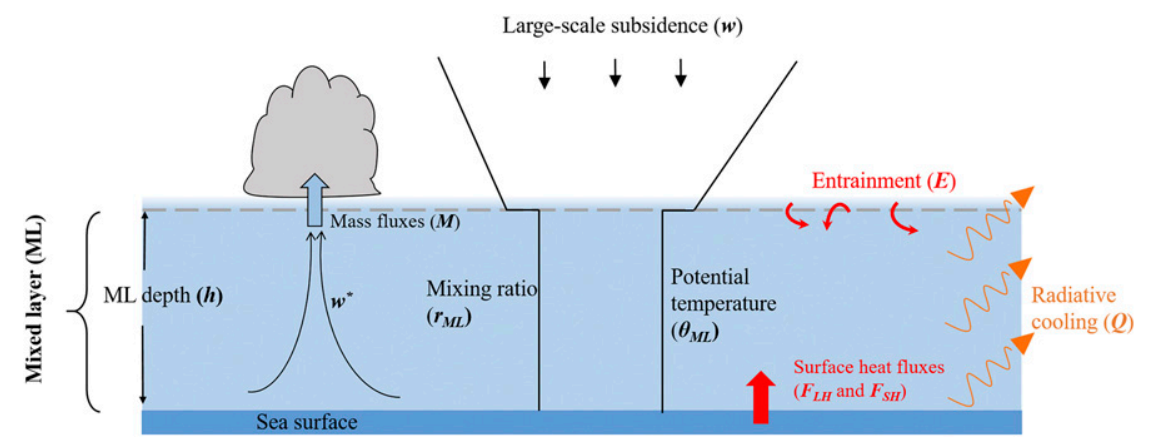

FIG. 1. A cartoon illustrating physical processes to be considered in the analytic mixedlayer model.

$$
w^{*}=\left(\frac{g h \overline{\theta_{v}}}{\left[w^{\prime} \theta_{v}^{\prime}\right]_{s}}\right)^{1 / 3},
$$

where $g$ is the acceleration of gravity, $h$ is the ML height, $\theta_{v}$ is the ML mean virtual potential temperature, and $\overline{\left[w^{\prime} \theta_{v}^{\prime}\right]_{s}}$ is the vertical kinetic heat flux near the surface. As discussed in the introduction, in an equilibrium shallow-cumulus-topped ML, it is tenable to use the $h$ as a proxy for $z_{b}$ (Betts 1973). The dependence of $w^{*}$ on the $h$ could be expanded by the chain rule:

$$
\frac{d w^{*}}{d h}=\frac{\partial w^{*}}{\partial h}+\frac{\partial w^{*}}{\partial \overline{\left[w^{\prime} \theta_{v}^{\prime}\right]_{s}}} \frac{\partial \overline{\left[w^{\prime} \theta_{v}^{\prime}\right]_{s}}}{\partial h}+\frac{\partial w^{*}}{\partial \theta_{v}} \frac{\partial \theta_{v}}{\partial h} .
$$

Here we focus on the first two terms on the right-hand side because the third term is at least one magnitude smaller than the other two (see appendix A for detail derivation). The first term on the right-hand side represents the direct dependence of updrafts on the $h\left(\lambda_{h}\right)$. According to Eq. (1), everything else equal, the equilibrium updrafts are stronger in a deeper ML. Physically speaking, if we consider a well-mixed state as an equilibrium state maintained by the exchanges between the turbulent kinetic energy and mean flow potential energy, a deeper ML contains a greater amount of air mass, which requires greater turbulence energy to maintain a well-mixed state.

The second term represents the dependence of updrafts on the $h$ through the feedback of the surface buoyancy fluxes $\lambda_{\mathrm{SBF}}$. To determine the $\partial\left[w^{\prime} \theta_{v}^{\prime}\right]_{s} / \partial h$, we use the budget equation of $\theta_{v}$ for a steady-state ML $\left(d \theta_{v} / d t=0\right)$ :

$$
\frac{\partial \overline{w^{\prime} \theta_{v}^{\prime}}}{\partial z}=-Q_{\mathrm{rad}}
$$

where $Q_{\text {rad }}$ is the radiative cooling rate (positive for cooling). Here, we assume that the convergence of buoyancy fluxes, to a zero order, balance the radiative cooling (Bretherton 1993; Nuijens and Stevens 2012; Schalkwijk et al. 2013). Integrating both sides from the surface to the ML top gives

$$
\overline{\left[w^{\prime} \theta_{v}^{\prime}\right]_{h}}-\overline{\left[w^{\prime} \theta_{v}^{\prime}\right]_{s}}=-h \widehat{Q_{\mathrm{rad}}},
$$

where the $\widehat{Q_{\text {rad }}}$ represents the vertically averaged $Q_{\text {rad }}$, and for clarity we use the $Q$ instead through the rest of the manuscript. The $\overline{\left[w^{\prime} \theta_{v}^{\prime}\right]_{h}}$ is typically a certain fraction, $a$, of $\overline{\left[w^{\prime} \theta_{v}^{\prime}\right]_{s}}$ (Ball 1960; Betts 1973; Stevens 2007). Experiments and large-eddy simulations show that $a=$ -0.2 is a common reference. With $\overline{\left[w^{\prime} \theta_{v}^{\prime}\right]_{h}}=\overline{a\left[w^{\prime} \theta_{v}^{\prime}\right]_{s}}$, Eq. (4) becomes

$$
\overline{\left[w^{\prime} \theta_{v}^{\prime}\right]_{s}}=\frac{Q}{1-a} h .
$$

Equation (5) indicates that given fixed entrainment efficiency and $Q$, a deeper ML is associated with stronger surface buoyancy fluxes. This makes physical sense because a deeper ML undergoes stronger radiative cooling, which requires stronger surface buoyancy fluxes to balance it.

Combining Eq. (5) with Eq. (1), we obtain the $\lambda_{h}$ and $\lambda_{\mathrm{SBF}}$ :

$$
\lambda_{h}=\lambda_{\mathrm{SBF}}=\frac{1}{3}\left(\frac{g}{\theta_{v}} \frac{Q}{1-a}\right)^{1 / 3} h^{-1 / 3} .
$$

It is interesting that these two terms are identical. More importantly, there is a dependence of $\lambda_{h}$ and $\lambda_{\mathrm{SBF}}$ on $h$, suggesting that the response of $w^{*}$ to $h$ is not strictly linear. Figure 2 shows the variations of $d w^{*} / d h$ and its components with the $h$ for given values of $a=-0.2$ and $Q=2 \mathrm{Kday}^{-1}$. A considerable degree of sublinearity is noticed when the $h$ is low. As $h$ increases, $d w^{*} / d h$ drops by a decreasing degree. Within a reasonable range of $h(300-1000 \mathrm{~m})$ for typical maritime cumulus clouds, $d w^{*} / d h$ varies rather little $\left(0.6-0.9 \mathrm{~m} \mathrm{~s}^{-1} \mathrm{~km}^{-1}\right)$. Such a limited variation of $d w^{*} / d h$ allows the relationship to appear linear. 


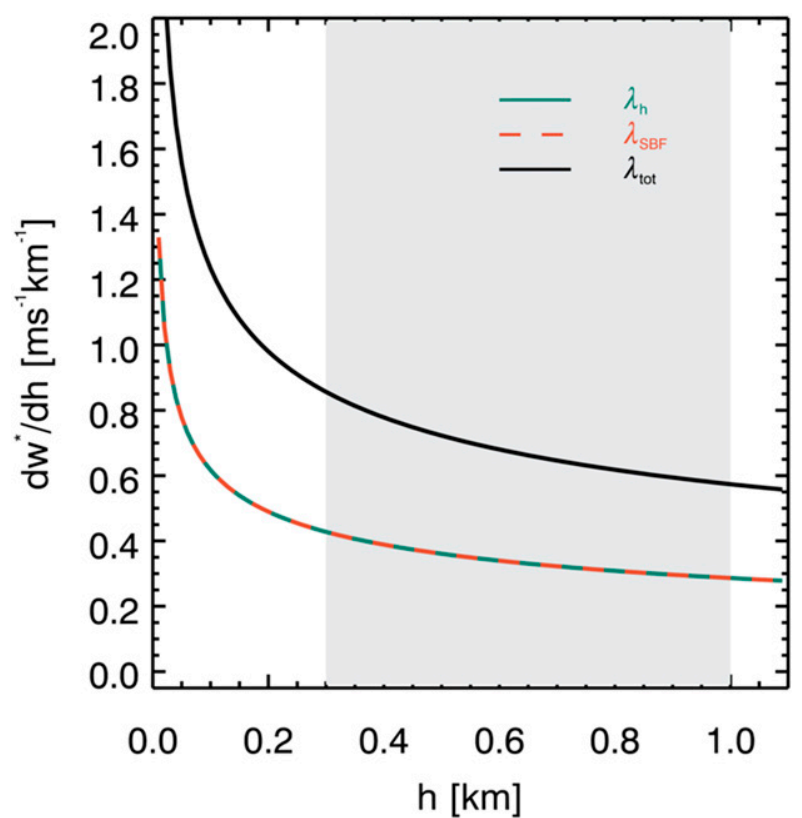

FIG. 2. Variations of the $d w^{*} / d h\left(\lambda_{\text {tot }}\right)$ and its components ( $\lambda_{h}$ and $\lambda_{\mathrm{SBF}}$ ) with the mixed-layer height $h$. The $\lambda_{\theta}$ is ignored in $\lambda_{\text {tot }}$ because of its smaller magnitude. The shaded region marked the normal range of $h$ for typical maritime boundary layers.

To fully understand the linearity, a critical question has to be asked: Is it tenable to consider $Q$ and $a$ as given? Figure 3 shows $d w^{*} / d h$ as a function of $h$ for different values of $Q$ and $a$. Both parameters influence $d w^{*} / d h$. At a given $h$, a higher $Q$ corresponds to stronger updrafts because a stronger cooling requires stronger surface buoyancy fluxes to balance it. If the entrainment efficiency is higher (smaller $a$ ), less surface buoyancy fluxes are required because the enhanced entrainment warming counteracts a certain fraction of radiative cooling.

In the remainder of this manuscript, we focus on $Q$ and its role in influencing the linearity. We choose $Q$ over $a$ for the following three reasons. First, as suggested by Fig. 3, $d w^{*} / d h$ is markedly more sensitive to $Q$ than to $a$ under the same degree of perturbation $( \pm 50 \%)$. Second, compared with $a$ that proves resistant to analytical treatment, $Q$ could be understood via basic radiative transfer equations. Third, while the analytical exploration of the radiative cooling for Earth's atmosphere as a whole is common (Doherty and Newell 1984; Weaver and Ramanathan 1995; Takahashi 2009; Robinson and Catling 2012; Beucler and Cronin 2016; Cronin and Jansen 2016), studies of this kind with a focus on the lower atmosphere (or ML) remain scant. Very often, an empirical value (e.g., $2 \mathrm{~K} \mathrm{day}^{-1}$ ) of $Q$ is specified in ML models (Neggers et al. 2006; Stevens 2006, 2007) and LES (Nuijens and Stevens 2012; Sakradzija and Hohenegger 2017). The physical reasons for fixing $Q$ and for selecting this specific value of $2 \mathrm{~K} \mathrm{day}^{-1}$, however, are lacking in direct evidence. By arbitrarily specifying a wide range of $Q$, a recent study stresses the significant role of $Q$ in modulating the dynamics of ML (Naumann et al. 2017). However, as a feedback, how $Q$ responds to changes in internal (e.g., $h$ and moisture loading) and external (e.g., free-tropospheric moisture) parameters of the ML system is certainly important. In the next section, I will use analytic radiative transfer formulas to understand the mechanism of ML radiative cooling rate, which will be used, in combination with an ML model, to quantify the sensitivity of $Q$ to changes in internal and external parameters.
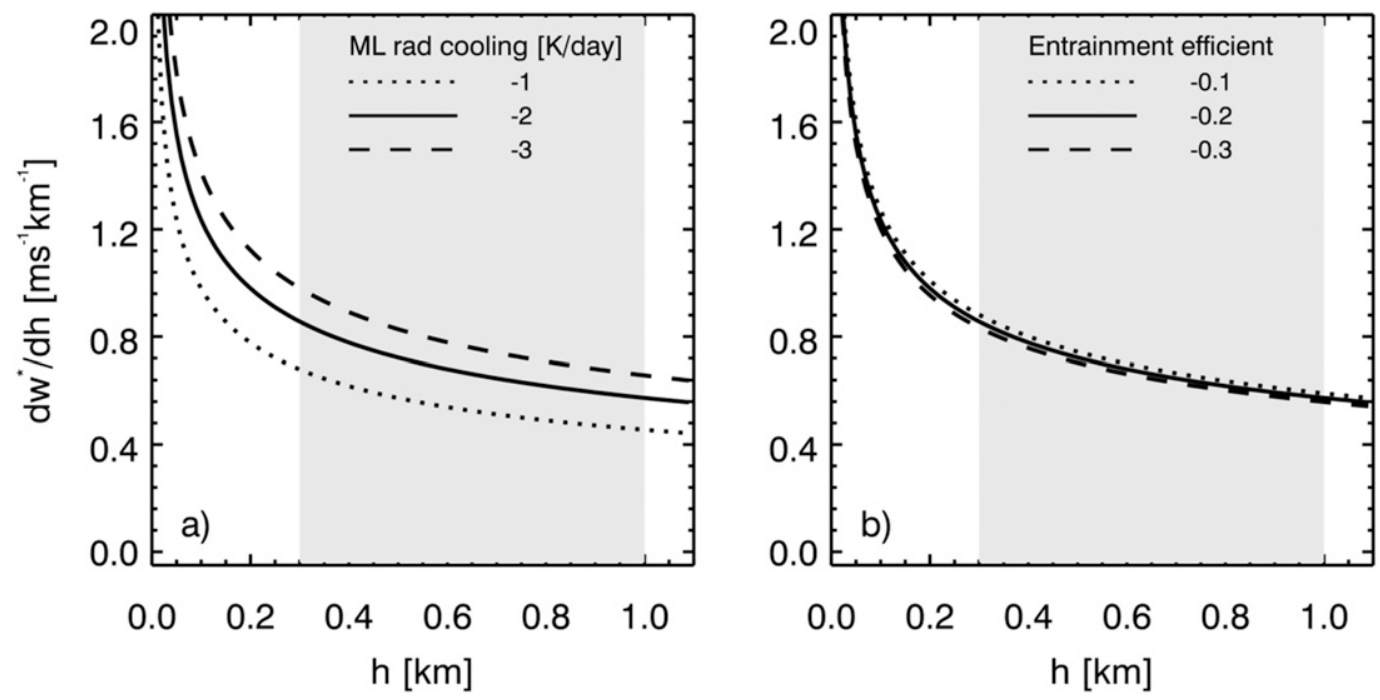

FIG. 3. Variations of $\lambda_{h}$ (or equally $\lambda_{\mathrm{SBF}}$ ) with the mixed-layer height $h$ for different values of (a) $Q$ and (b) $a$. 
a) Temperature profile

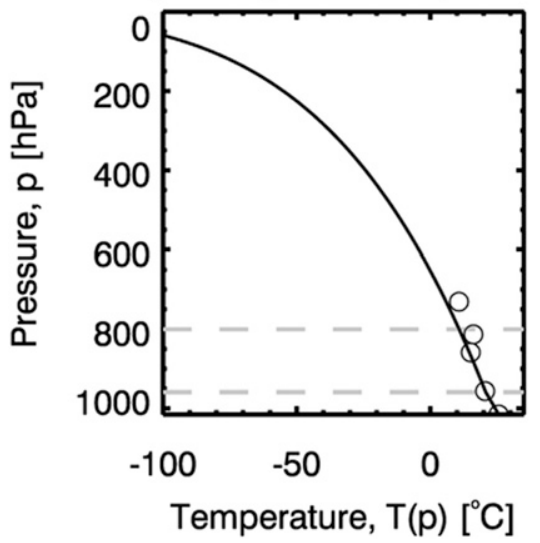

b) Mixing ratio profile

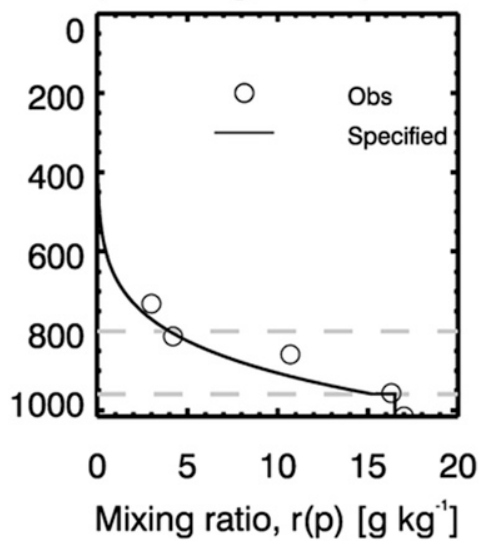

c) Mixing ratio profile (log)

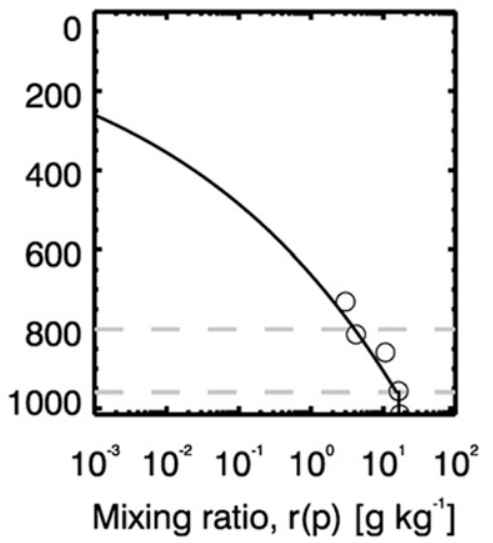

FIG. 4. Vertical profiles of (a) temperature and water vapor mixing ratio in (b) linear and (c) log scales. The lines and the open circles stand for the fitted profile and the aircraft measurements during the BOMEX field experiment, respectively. The two dashed gray lines mark the altitudes of $800 \mathrm{hPa}$ and the ML top.

\section{Analytic understanding of the mixed-layer radiative cooling}

The main objective in this section is to establish an intuition-based understanding of the ML radiative cooling rate. This is the reason why, instead of relying on full-radiation code, we use analytic formulas that are heuristic and analytically more tractable. It is particularly necessary for the longwave radiative cooling, to understand which is more nontrivial than its shortwave counterpart. The analytic calculations will be validated against those from full-radiation code.

\section{a. Temperature and moisture profile}

A typical atmospheric sounding associated with shallow cumulus clouds is characterized by a wellmixed subcloud layer, a shallow transition layer near the cloud base, a conditionally unstable cloudy layer topped by an inversion layer, and a free-atmosphere (FA) layer. An accurate specification of this structure complicates the algebra, which defeats the heuristic purpose. Our strategy is to prescribe atmospheric profiles that are simple enough for heuristic understanding without losing an authentic representation of the real atmosphere. Figure 4 shows an example of the specified temperature and water vapor mixing ratio profiles for a shallow cumulus case during the Barbados Oceanographic and Meteorological Experiment (BOMEX) field campaign (Holland and Rasmusson 1973; Siebesma et al. 2003). The open circles mark the aircraft measurements. We fit the profiles by considering the atmosphere as a two-layer structure: a wellmixed subcloud layer with constant water vapor mixing ratio and potential temperature and an FA with the mixing ratio and temperature distributed in power-law form:

$T(p)=T_{s} \times\left\{\begin{array}{cc}\left(\frac{p}{p_{s}}\right)^{R_{d} / c_{p}}, & \text { for } p>p_{h} \\ \left(\frac{p_{h}}{p_{s}}\right)^{R_{d} / c_{p}}\left(\frac{p}{p_{h}}\right)^{R_{d} \Gamma / g}, & \text { for } p \leq p_{h}\end{array}\right.$,

$r(p)=\left\{\begin{array}{ccc}r_{\mathrm{ML}}, & \text { for } & p>p_{h} \\ \left(r_{\mathrm{ML}}+\Delta r\right)\left(\frac{p}{p_{h}}\right)^{n}, & \text { for } & p \leq p_{h}\end{array}\right.$,

where $T, r$, and $p$ are temperature, water vapor mixing ratio, and pressure, respectively, subscripts $s$ and $h$ represent the properties at the surface and the ML top, respectively; $\Gamma=6.5 \mathrm{~K} \mathrm{~km}^{-1}$ is the lapse rate of the FA potential temperature; and $n$ stands for the rate with which the FA moisture decreases with the altitude. The temperature first decreases with height adiabatically and then in power law in the FA. Here we ignore the temperature inversion, which may lead to a slight underestimation of the FA temperature, as is shown in the Fig. 4a. Given that the temperature profile is much less influential in thermal radiative cooling than the water vapor (Mapes and Zuidema 1996), this simplification is tenable.

The water vapor mixing ratio profile is specified in a similar way, but we account for a moist inversion $\Delta r$ at the top of the ML. This is necessary because the longwave radiative cooling rate is most sensitive to the vertical gradient of thermal transmission that is largely 
controlled by moisture loading. The value of $\Delta r$ is parameterized by an empirical relationship:

$$
\Delta r=C_{r} \times\left[r_{\mathrm{ML}}-r(p=800 \mathrm{hPa})\right],
$$

where the $C_{r}$ is the coefficient representing the efficiency of transporting free-tropospheric air downward through the cloud layer to the ML top. We use the empirical value of 0.1 determined by Neggers et al. (2006). This parameterization allows the $n$ to be uniquely determined if the $p_{h}, r_{\mathrm{ML}}$, and $r$ at $800 \mathrm{hPa}$ $\left(r_{800}\right)$ are given.

The simplified two-layer structure of $r$ does not account for the cloud layer that lies between the ML and FA. This may cause an underestimation of $r$ for the atmosphere just overlying the ML top (as visible in Figs. $4 \mathrm{~b}$ and $4 \mathrm{c}$ ). The radiative consequence of this simplification will be discussed in section $4 \mathrm{~b}$ in which $Q$ of the idealized cases will be compared with the realworld cases. Moreover, for simplicity, we do not consider the clouds themselves as effective emitters because the cloud amount of shallow cumulus $(<30 \%)$ is not adequately large for markedly influencing the ML radiative budget as far as the domain-integrated radiative cooling is concerned. In principle, the cloud layer, on one hand, warms the ML by greenhouse effects, and, on the other hand, cools the ML by blocking the incoming solar radiation. As a result, the combined effect is at most moderate because of the counteraction.

\section{b. Radiative transfer theory}

We consider a plane-parallel atmosphere with water vapor as the only absorber and emitter. A gray atmosphere is assumed so that there is no dependence of thermal absorption on wavelength. We use the average water vapor absorption coefficient $k_{\mathrm{LW}}=0.1 \mathrm{~m}^{2} \mathrm{~kg}^{-1}$ (Beucler and Cronin 2016). The profile of optical thickness $\tau$ could be described by

$$
\tau(p)=D \int_{0}^{p} K(p) \frac{d p}{g},
$$

where $D=5 / 3$ is the diffusivity factor that accounts for the hemisphere-integrated radiances, and $K(p)$ is the absorption coefficient for the atmosphere. Since the water vapor is the only absorber, $K(p)=k_{\mathrm{Lw}} r(p)$. Following Beucler and Cronin (2016), we multiply the $K(p)$ by $p / p_{s}$ to account for the pressure broadening of water vapor.

The ML longwave radiative fluxes divergence $\Delta F_{\mathrm{LW}}$ could be written as

$$
\Delta F_{\mathrm{LW}}=F_{\uparrow}\left(\tau_{h}\right)-F_{\downarrow}\left(\tau_{h}\right)-\left[F_{\uparrow}\left(\tau_{s}\right)-F_{\downarrow}\left(\tau_{s}\right)\right],
$$

where the $F_{\uparrow}$ and $F_{\downarrow}$ represent the upward and downward radiative fluxes, respectively. To calculate the radiative fluxes, we start with the two-stream Schwarzschild equations:

$$
\begin{aligned}
& \frac{d F_{\uparrow}}{d \tau}=F_{\uparrow}-\sigma T^{4}, \\
& \frac{d F_{\downarrow}}{d \tau}=-\left(F_{\downarrow}-\sigma T^{4}\right),
\end{aligned}
$$

in which the $\sigma$ is the Boltzmann constant. With the boundary condition of $F_{\uparrow}\left(\tau=\tau_{s}\right)=\sigma T^{4}$, we integrate the Eq. (11a) from $\tau^{\prime}=\tau$ to $\tau^{\prime}=\tau_{s}$ and obtain the $F_{\uparrow}$ profile. Likewise, the downward radiative fluxes profile could be obtained by integrating the Eq. (11b) from $\tau^{\prime}=$ 0 to $\tau^{\prime}=\tau$ with the boundary condition of $F_{\downarrow}(\tau=0)=0$. The readers can refer to Robinson and Catling (2012) for detailed derivation. With the derived profiles of $F_{\uparrow}$ and $F_{\downarrow}, \Delta F_{\mathrm{LW}}$ scaled by $\sigma T_{s}^{4}$ is expressed as

$$
\begin{aligned}
\frac{\Delta F_{\mathrm{LW}}}{\sigma T_{s}^{4}}= & \int_{\tau_{h}}^{\tau_{s}} d \tau^{\prime} B_{\mathrm{ML}}\left(\tau^{\prime}\right) e^{-\left(\tau_{s}-\tau^{\prime}\right)}-\left[1-e^{-\left(\tau_{s}-\tau_{h}\right)}\right] \\
& +\int_{\tau_{h}}^{\tau_{s}} d \tau^{\prime} B_{\mathrm{ML}}\left(\tau^{\prime}\right) e^{-\left(\tau^{\prime}-\tau_{h}\right)}\left(1-e^{-\tau_{h}}\right) \\
& -\int_{0}^{\tau_{h}} d \tau^{\prime} B_{\mathrm{FA}}\left(\tau^{\prime}\right) e^{-\left(\tau_{h}-\tau^{\prime}\right)}\left[1-e^{-\left(\tau_{s}-\tau_{h}\right)}\right] \\
& +\int_{\tau_{h}}^{\tau_{s}} d \tau^{\prime} B_{\mathrm{ML}}\left(\tau^{\prime}\right) e^{-\tau^{\prime}}
\end{aligned}
$$

where the $B(\tau)=\sigma T(\tau)^{4} / \sigma T_{s}^{4}$ and the subscripts "FA" and "ML" represent the free atmosphere and mixed layer, respectively. See appendix B for detailed derivation of Eq. (12). The physical meanings of the three terms on the right-hand side in sequence are

1) exchange of radiative energy with the surface,

2) exchange of radiative energy with FA, and

3) cooling to space.

The first term and second term warms and cools the ML, respectively, because of the warmer-and-moister surface and colder-and-drier FA. This counteracting effect leaves the "cooling to space" term the dominant factor regulating the cooling rate, which is commonly called the cooling-to-space approximation (Mapes and Zuidema 1996; Petty 2006). This approximation, if tenable, can help simplify the equation, allowing us to understand the longwave radiative cooling process in a more intuitive way. We will further test this idea in the 
a) Temperature profile

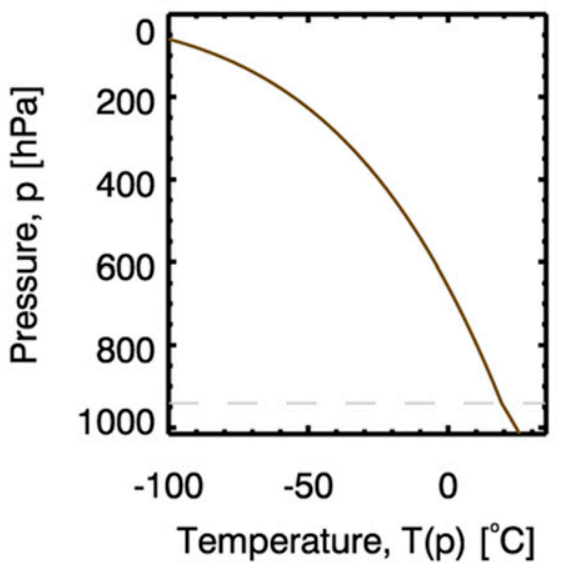

b) Mixing ratio profile

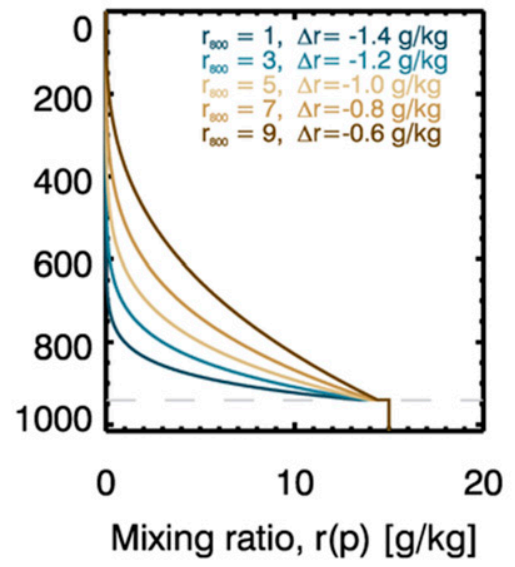

c) $\tau$ profile

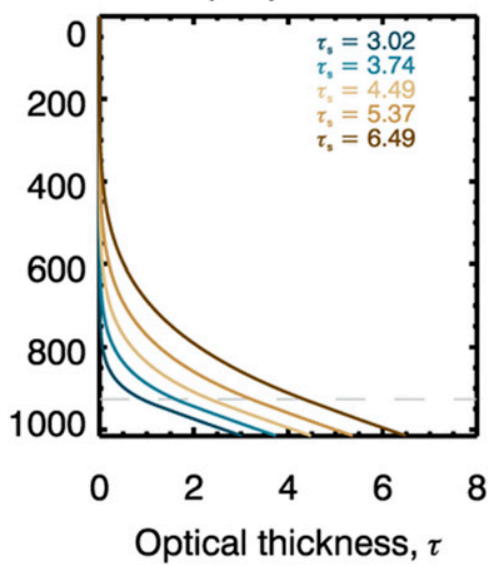

FIG. 5. Vertical profiles of specified (a) temperature, (b) water vapor mixing ratio, and (c) thermal optical thickness. The gray dashed line marks the ML height $h$.

next section under reasonable thermodynamic settings typical of shallow cumulus regimes.

\section{c. Analytical exploration}

\section{1) VARIED FREE-ATMOSPHERE MOISTURE}

We use the analytic equations from section $3 b$ to explore the sensitivity of $\Delta F_{\mathrm{LW}}$ to different thermodynamic settings of the atmosphere. An appropriate starting point is to examine the effect of FA moisture loading that has been widely regarded as the dominant factor controlling $\Delta F_{\mathrm{LW}}$ (Mapes and Zuidema 1996; Wood 2012; Stevens et al. 2017). To isolate the radiative role of FA moisture, we fix the ML mixing ratio and ML height. By varying the $r_{800}$ from 1 to $9 \mathrm{~g} \mathrm{~kg}^{-1}$ (Fig. 5b), we generate a series of atmospheric soundings spanning a spectrum of FA opaqueness (Fig. 5c). The sensitivity of the analytically computed $\Delta F_{\text {LW }}$ to the FA moisture is shown in Fig. 6a in which we use the FA precipitable water to characterize the moisture burden. A considerable degree of sensitivity is noted. The ML air cools more significantly when FA is drier. Analysis of each component in Eq. (12) shows a dominant role of cooling-to-space term in controlling the cooling. A drier FA allows for a more exposure of the ML air to cold space, enhancing $\Delta F_{\mathrm{LW}}$. The "exchange with the free atmosphere" term also causes cooling, the intensity of which increases with the FA moisture burden. Physically, the ML air loses more energy to FA as the FA moisture burden increases. The rate with which the cooling increases with the FA moisture is much less dramatic than the decreasing rate of the cooling-to-space term.

To validate the analytic calculations, we use the Santa Barbara Discreet Ordinate Radiative Transfer (DISORT) Atmospheric Radiative Transfer (SBDART) model
(Ricchiazzi et al. 1998) to calculate $\Delta F_{\mathrm{LW}}$. The greenhouse gas loadings are set to their default values with $\mathrm{CO}_{2}$ concentration of $380 \mathrm{ppv}$. There are 65 vertical levels with grid intervals of $50 \mathrm{~m}$ in the lowest $1800 \mathrm{~m}$, $500 \mathrm{~m}$ between 1800 and $15000 \mathrm{~m}$, and $3000 \mathrm{~m}$ for altitudes greater than $15000 \mathrm{~m}$.
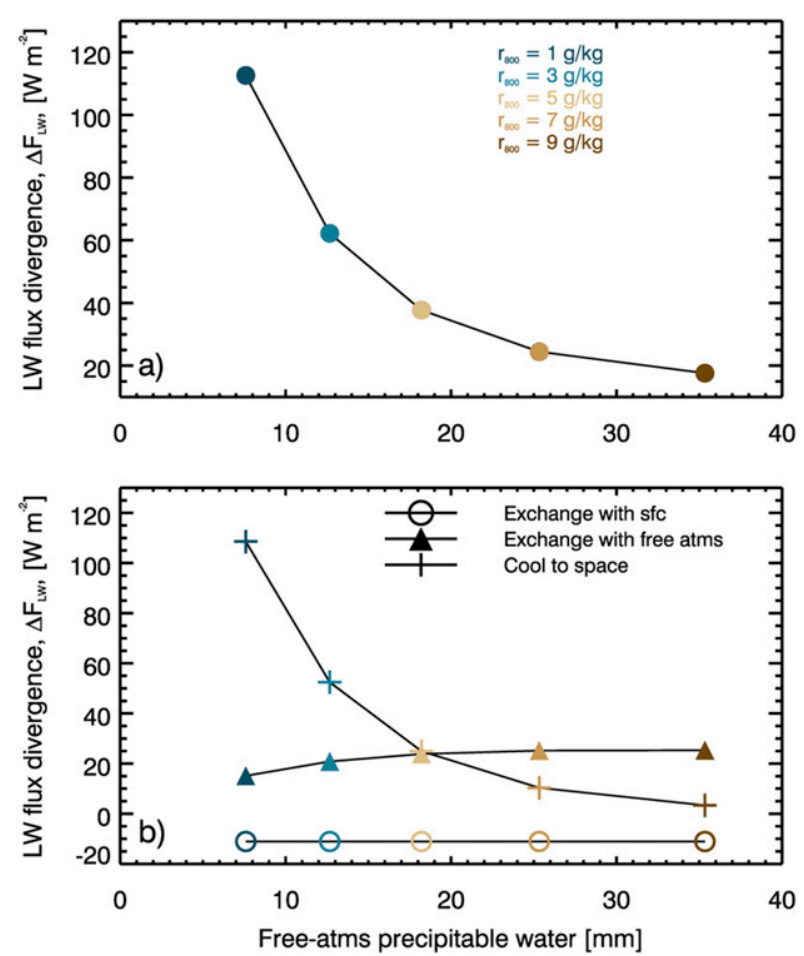

FIG. 6. Variations of (a) $\Delta F_{\mathrm{LW}}$ and (b) its components with the freeatmospheric precipitable water. 


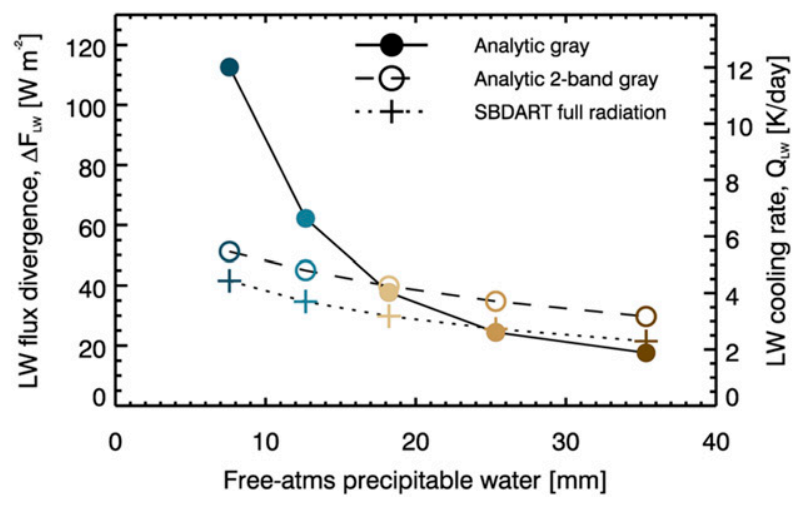

FIG. 7. $\Delta F_{\text {LW }}$ calculated analytically and by the SBDART fullradiation code as a function of the free-tropospheric precipitable water.

Comparison between the analytic solutions (filled circles) and the full-radiation calculations (plus signs) shows qualitatively consistent but quantitatively distinct results (Fig. 7). The analytic calculation significantly overestimates $\Delta F_{\mathrm{LW}}$ when the FA is dry. Such an overestimation is largely due to the fact that the gray-atmosphere assumption becomes increasingly less valid as the FA moisture drops. When the FA water vapor loading is low, ignoring the wavelength dependence (e.g., strongly absorbing water vapor bands) leads to an extremely transparent FA. Such a behavior is not realistic because in the real atmosphere the existence of the strong-absorption band of water vapor, along with the greenhouse gases, buffers the dryness-induced transparency. To examine this idea, we, by following Beucler and Cronin (2016), consider an atmosphere with two absorption bands of water vapor: a strong-absorption band $\left(k_{\mathrm{LW}}=\right.$ $\left.1 \mathrm{~m}^{2} \mathrm{~kg}^{-1}\right)$ and a weak-absorption band $\left(k_{\mathrm{LW}}=0.02 \mathrm{~m}^{2} \mathrm{~kg}^{-1}\right)$. Assuming that the two bands occupy half of the water vapor absorption spectrum, the total $\Delta F_{\mathrm{LW}}$ is simply an average of $\Delta F_{\mathrm{LW}}$ for the two bands. Compared with the single-band calculation, the two-band calculation (open circles in Fig. 7) shows a result more consistent with the fullradiation computation despite a systematic overestimation of $\Delta F_{\mathrm{LW}}$ by $\sim 10 \mathrm{~W} \mathrm{~m}^{-2}$. Such an overestimation may arise from the warming effects of greenhouse gases that are not accounted for in the analytic equations. The strongabsorption band prevents the ML from cooling too dramatically as the FA dries. This allows for a relatively short variation of $Q_{\mathrm{LW}}\left(2-3.5 \mathrm{~K} \mathrm{day}^{-1}\right)$ across the wide spectrum of FA moisture burden. As will be shown later, the dynamical impacts of the FA moisture on the entrainment process further limit the variation of $Q_{\mathrm{LW}}$.

\section{2) VARIED ML MOISTURE AND DEPTH}

In addition to FA moisture that is considered as an external variable of an ML system, the internal parameters such as $r_{\mathrm{ML}}$ and $h$ should also influence $Q_{\mathrm{LW}}$. These two parameters typically covary. For example, an increase in SST, via enhancing the entrainment rate, can both deepen and desiccate the ML (Bretherton and Wyant 1997). When an ML is topped by cumulus clouds, the proxy between $h$ and LCL, as an emergent constraint, requires $h$ to decrease with increase in $r_{\mathrm{ML}}$ (Betts 1976; Albrecht et al. 1979; Schalkwijk et al. 2013). Therefore, a prototype question that is helpful for understanding real-world cumulus-topped MLs is, How is $Q_{\mathrm{LW}}$ regulated by the covarying $r_{\mathrm{ML}}$ and $h$ ? To that end, we vary $r_{\mathrm{ML}}$ and $h$ using the analytic formula of LCL as a function of humidity (Romps 2017) while maintaining other parameters (including $\theta_{\mathrm{ML}}=298 \mathrm{~K}$ and $r_{800}=$ $5 \mathrm{~g} \mathrm{~kg}^{-1}$ ) unchanged (Fig. 8a). By so doing, two sources of complexity emerge. First, the ML precipitable water a) Temperature profile

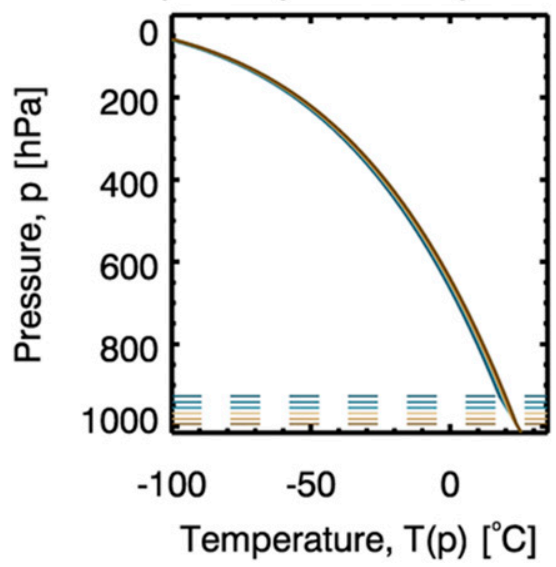

b) Mixing ratio profile

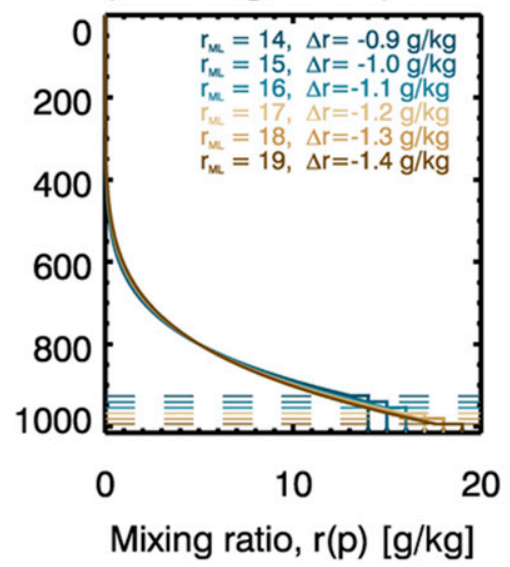

c) $\tau$ profile

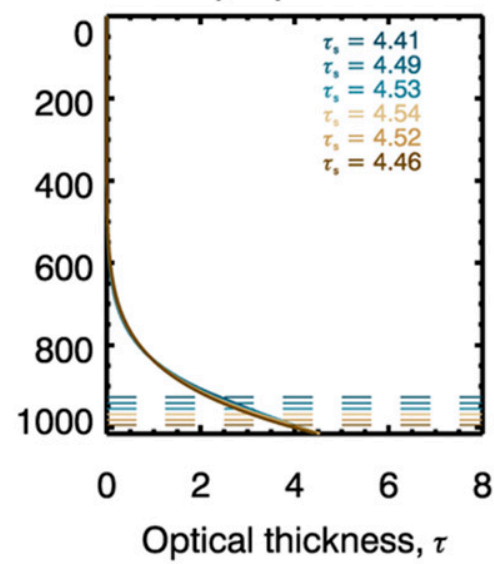

FIG. 8. Vertical profiles of specified (a) temperature, (b) water vapor mixing ratio, and (c) thermal optical thickness. The dashed lines mark the ML top height $h$. 


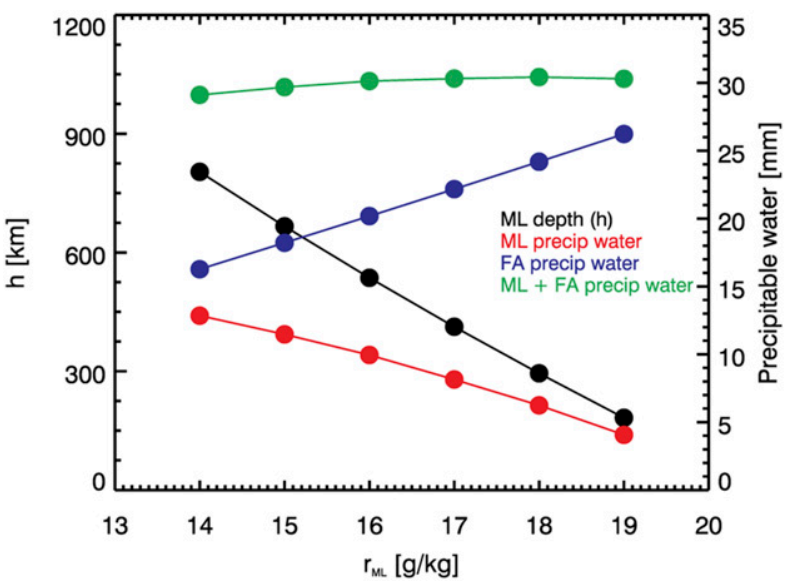

FIG. 9. Variations of $h$ (black), ML precipitable water (red), FA precipitable water (blue), and total precipitable water (green) as a function of $r_{\mathrm{ML}}$

(or the opaqueness of the ML) decreases markedly with an increase in $r_{\mathrm{ML}}$ because the ML becomes shallower (Fig. 9). The total precipitable water of the atmosphere, however, remains nearly invariant (Fig. 9). This invariance allows the FA precipitable water to increases with $r_{\mathrm{ML}}$ (Fig. 9). This means that despite $r_{800}$ is held constant, the FA moisture loading still changes. Second, since $h$ varies, the variations of $\Delta F_{\mathrm{LW}}$ and $Q_{\mathrm{LW}}$ can no longer be considered simultaneously because of their dependence on $h$ via $Q_{\mathrm{LW}}=\Delta F_{\mathrm{LW}} / c_{p} \rho h$.

Figure $10 \mathrm{a}$ shows that as $r_{\mathrm{ML}}$ increases, $\Delta F_{\mathrm{LW}}$ and $Q_{\mathrm{LW}}$ decreases and increases, respectively. It is nontrivial to explain these relationships with the full version of Eq. (12). A simplification is needed. By examining the contributions of the three components, we found that the cooling-to-space approximation is a good proxy for the net radiative cooling. The exchanges of heat with the surface and with the FA warm and cool the ML air, respectively, by a comparable degree. Such a cancellation renders the cooling-tospace term dominant in determining $\Delta F_{\mathrm{LW}}$ and $Q_{\mathrm{LW}}$. To examine the universality of the cooling-to-space approximation, we systematically compare the cooling-tospace components $\Delta F_{\mathrm{LW}}^{\mathrm{CTS}}$ and $Q_{\mathrm{LW}}^{\mathrm{CTS}}$ with the full-equation results for all the cases (Fig. 11). The agreements are excellent.

Having confirmed the validity of the cooling-to-space approximation, we further simplify Eq. (12):

$$
\begin{aligned}
\frac{\Delta F_{\mathrm{LW}}}{\sigma T_{s}^{4}} & \approx \frac{\Delta F_{\mathrm{LW}}^{\mathrm{CTS}}}{\sigma T_{s}^{4}} \\
& =\int_{\tau_{h}}^{\tau_{s}} d \tau^{\prime} B\left(\tau^{\prime}\right) e^{-\tau^{\prime}} \approx \Delta \tau_{\mathrm{ML}}\left(r_{\mathrm{ML}}, h\right) B\left(\widehat{\tau_{\mathrm{ML}}}\right) e^{-\widehat{\tau_{\mathrm{ML}}}},
\end{aligned}
$$
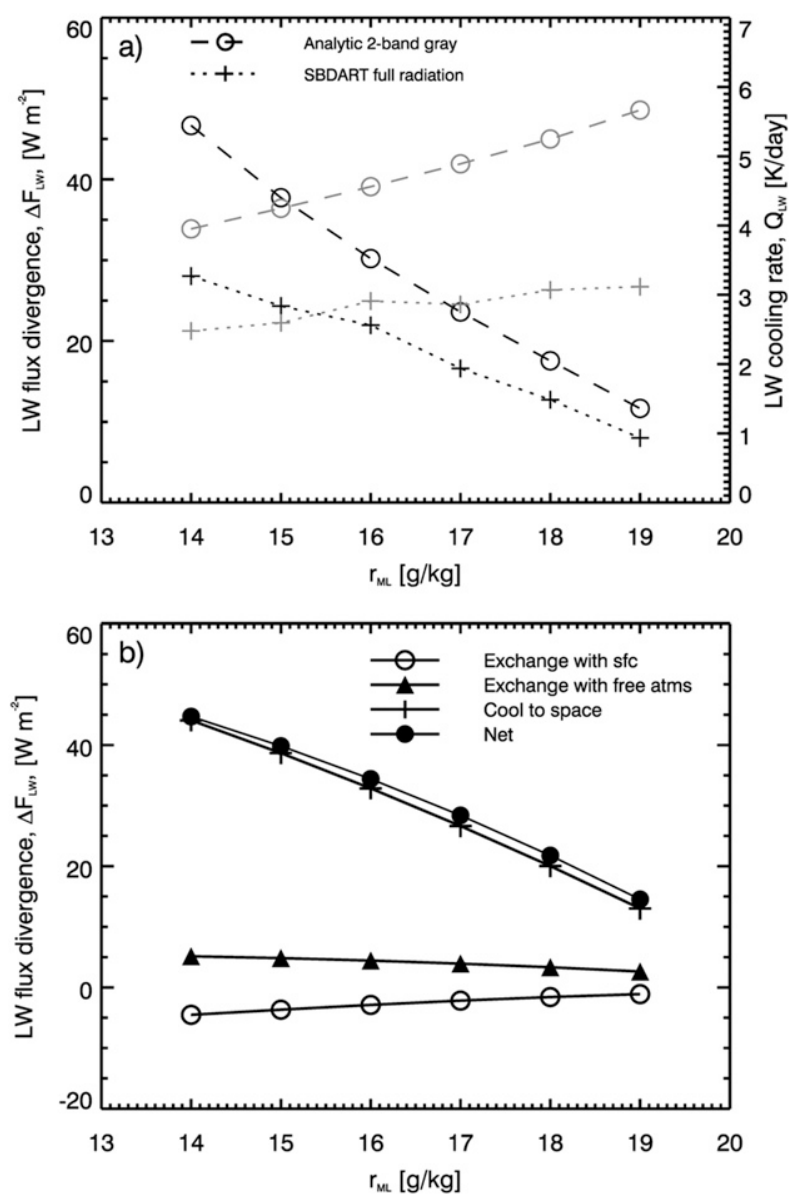

FIG. 10. (a) $\Delta F_{\mathrm{LW}}$ (black) and $Q_{\mathrm{LW}}$ (gray) calculated by two-band analytic formulas and full-radiation code as a function of ML water vapor mixing ratio $r_{\mathrm{ML}}$. (b) The components of two-band analytic computations of $\Delta F_{\mathrm{LW}}$ as a function of $r_{\mathrm{ML}}$.

where the $\Delta \tau_{\mathrm{ML}}=\tau_{s}-\tau_{h}$, and $\widehat{\tau_{\mathrm{ML}}}$ represents the vertically averaged optical depth in the ML. Physically, the $\Delta \tau_{\mathrm{ML}}$ and $e^{-\widehat{\mathrm{TL}_{\mathrm{ML}}}}$ represent the emitter amount in the ML and the exposure of the ML air to space, respectively. Either greater emitter amount or greater exposure to the space causes stronger radiative cooling.

With this simplified framework, it becomes straightforward to understand why $\Delta F_{\mathrm{LW}}$ decreases with $r_{\mathrm{ML}}$. By increasing the $r_{\mathrm{ML}}$, the ML precipitable water and $\Delta \tau_{\mathrm{ML}}$ decrease (Fig. 8c). Since the $\tau_{s}$ remains nearly invariant (Fig. 8c), $\widehat{\tau_{\mathrm{ML}}}$ increases. Decreased $\Delta \tau_{\mathrm{ML}}$ and increased $\widehat{\tau_{\mathrm{ML}}}$ correspond to less emitter amount and less exposure to space, leading to lower $\Delta F_{\mathrm{LW}}$.

Such a simple formula could also be used to understand $Q_{\mathrm{LW}}$. The $Q_{\mathrm{LW}}$ is of more interest here because it directly determines the slope of the relationship between the updrafts and $h$ (Fig. 2). The approximate expression for $Q_{\mathrm{LW}}$ could be derived by dividing both sides of the Eq. (13) by $c_{p} \rho h$ : 

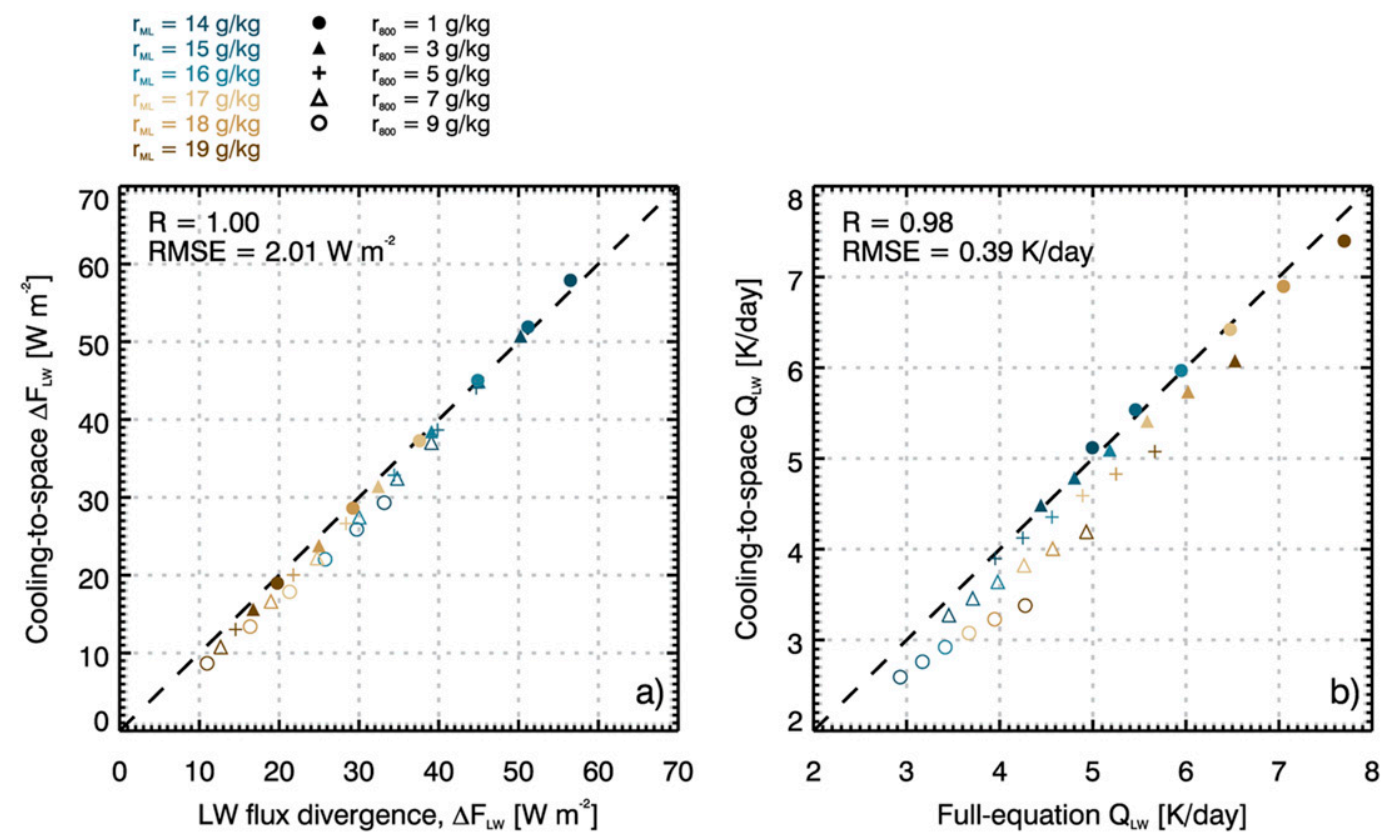

FIG. 11. Comparisons between the cooling-to-space component of analytic (a) $\Delta F_{\mathrm{LW}}$ and (b) $Q_{\mathrm{LW}}$ and those from the full equation. The RMSE stands for the root-mean-square error.

$$
\begin{aligned}
\frac{Q_{\mathrm{LW}}^{\mathrm{CTS}}}{\sigma T_{s}^{4}} & =\frac{\Delta \tau_{\mathrm{ML}}\left(r_{\mathrm{ML}}, h\right)}{c_{p} \rho h} B\left(\widehat{\tau_{\mathrm{ML}}}\right) e^{-\widehat{\tau_{\mathrm{ML}}}} \\
& =\frac{1}{c_{p} \rho} \delta \tau_{\mathrm{ML}}\left(r_{\mathrm{ML}}\right) B\left(\widehat{\tau_{\mathrm{ML}}}\right) e^{-\widehat{\tau_{\mathrm{ML}}}}
\end{aligned}
$$

The emitter amount term on the right-hand side of Eq. (14) now becomes independent of $h$. This means that increase in $r_{\mathrm{ML}}$ will increase the emitter amount per unit air mass, increasing $Q_{\mathrm{LW}}$. Both the full-radiation and two-band analytic calculation shows that the degree with which $Q_{\mathrm{LW}}$ increases with the emitter mount outweighs the decrease of $Q_{\mathrm{LW}}$ with the decrease in the radiative exposure, leading to a net increase in $Q_{\mathrm{LW}}$ with the $r_{\mathrm{ML}}$.

\section{3) A SIMPLE ANALYTIC EXPRESSION FOR ML RADIATIVE COOLING RATE}

The validity of the analytic two-band radiative transfer formulas and the cooling-to-space approximation allow us to develop a simple formula that parameterizes the $\mathrm{ML}$ radiative cooling rate. We compare the analytic $Q_{\mathrm{LW}}^{\mathrm{CTS}}$ with the SBDART-computed $Q_{\mathrm{LW}}$ in Fig. $12 \mathrm{a}$. They correlate with each other very well despite a systematic overestimation of $1.56 \pm 0.33 \mathrm{~K} \mathrm{day}^{-1}$ for $Q_{\mathrm{LW}}^{\mathrm{CTS}}$ primarily due to neglecting the warming effect of the greenhouse gases. We simply subtract the analytic $Q_{\mathrm{LW}}^{\mathrm{CTS}}$ by $1.56 \mathrm{~K}$ to roughly account for this effect.

To gain a full description of $Q$, the shortwave component $Q_{\text {sw }}$ is needed. We employ the parameterization of Mapes and Zuidema (1996): $Q_{\mathrm{Sw}}=-(1 / \pi) Q_{\mathrm{LW}}$. To validate the parameterized $Q_{\mathrm{Sw}}$, we run the SBDART code in a solar zenith angle of $\arccos (\pi / 4) \mathrm{rad}$, which is the equatorial equinox value. The simulated shortwave fluxes are multiplied by $4 / \pi^{2}$ (Beucler and Cronin 2016) to account for the diurnal cycle and the zenith angle. The parameterized $Q_{\mathrm{Sw}}$ is correlated well with the SBDART calculation (Fig. 12b). With $Q_{\mathrm{LW}}$ and $Q_{\mathrm{SW}}$, the net radiative cooling could be expressed simply as

$$
\begin{aligned}
Q= & \left(1-\frac{1}{\pi}\right)\left[\frac{Q_{\mathrm{LW}}^{\mathrm{CTS}}\left(k_{\mathrm{LW}}=0.02\right)+Q_{\mathrm{LW}}^{\mathrm{CTS}}\left(k_{\mathrm{LW}}=1\right)}{2}\right. \\
& -1.56] .
\end{aligned}
$$

Validation of the $Q$ calculated by Eq. (15) against the full-radiation calculation shows an excellent agreement (Fig. 12c).

\section{Mixed-layer model coupled with radiation}

The atmospheric sounding is continuously shaped by vertical replacement of air parcels at different levels. In a typical ML, changes in the moisture of the overlying FA air modify the buoyancy jump at the top of the ML, influencing the entrainment process and the consequent changes in the moisture and mass budget of the ML. To account for this dynamic process, we use the bulk model for shallow-cumulus-topped ML developed by 


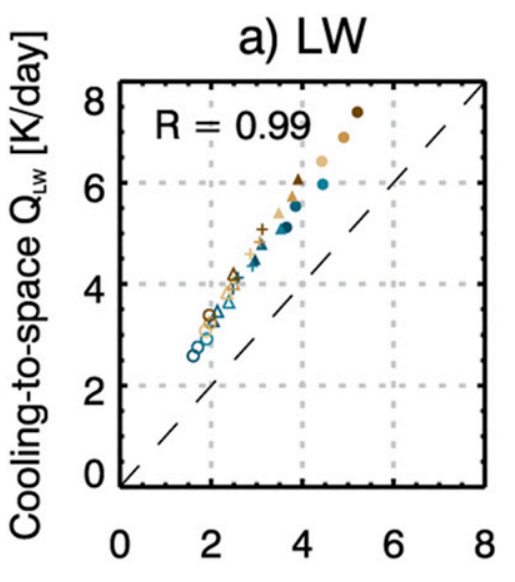

Full-radiation $Q_{L w}[K /$ day $]$

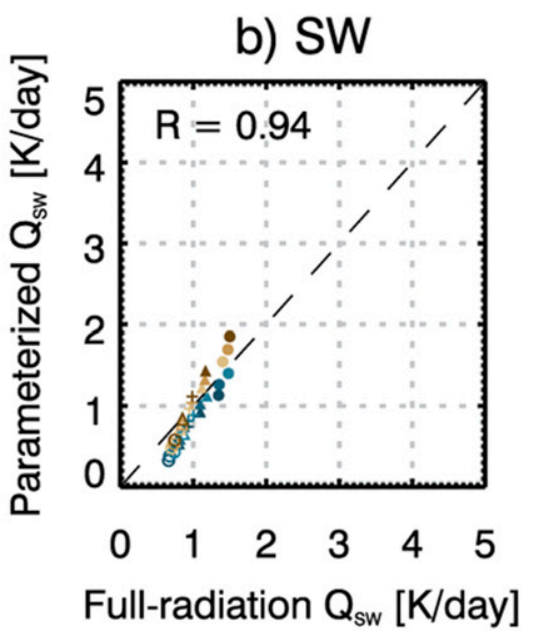

FIG. 12. Comparisons between the (a) cooling-to-space component of $Q_{\mathrm{LW}}$ and the SBDART $Q_{\mathrm{LW}}$, (b) parameterized $Q_{\mathrm{SW}}$ and the SBDART $Q_{\mathrm{SW}}$, and (c) parameterized $Q$ and SBDART $Q$.

Neggers et al. (2006) and couple it with the above-derived analytic formula for $Q$. Here, we give a brief description of the ML model. The model has three prognostic equations for $h, r_{\mathrm{ML}}$, and $\theta_{\mathrm{ML}}$, constrained by conservations of mass, moisture, and enthalpy, respectively:

$$
\begin{aligned}
\frac{\partial h}{\partial t} & =E+w-M, \\
h \frac{\partial r_{\mathrm{ML}}}{\partial t} & =F_{\mathrm{LH}}+E \Delta r+F_{\mathrm{adv}}^{r}, \\
h \frac{\partial \theta_{\mathrm{ML}}}{\partial t} & =F_{\mathrm{SH}}+E \Delta \theta-h Q+F_{\mathrm{adv}}^{\theta},
\end{aligned}
$$

where the $E, w$, and $M$ are the entrainment flux, largescale vertical velocity, and the mass fluxes of cumulus convection. The $F_{\text {adv }}^{\theta}$ and $F_{\text {adv }}^{r}$ terms are horizontal advection of $\theta_{\mathrm{ML}}$ and $r_{\mathrm{ML}}$, respectively. The $F_{\mathrm{SH}}$ and $F_{\mathrm{LH}}$ terms are the surface fluxes of sensible heat and water vapor mixing ratio, respectively. They are parameterized by

$$
\begin{aligned}
& F_{\mathrm{SH}}=0.0012 V\left(\theta_{s}-\theta_{\mathrm{ML}}\right), \\
& F_{\mathrm{LH}}=\alpha_{\mathrm{LH}} 0.0012 V\left(r_{s}-r_{\mathrm{ML}}\right),
\end{aligned}
$$

in which the $V$ is the surface wind speed and 0.0012 is the typical value of bulk transfer coefficient for the ocean surface (Garratt 1977; Stull 2012). Following Hansen and Back (2015), I insert an evaporative conductance parameter $\alpha_{\mathrm{LH}}$ in Eq. (17b) to alter the surface Bowen ratio. The value of $\alpha_{\mathrm{LH}}$ is initially set to 1 .

The entrainment velocity $E$ is parameterized by buoyancy fluxes at the ML top divided by the vertical jump of $\theta_{v}$ :

$$
E=-\frac{\left[w^{\prime} \theta_{v}^{\prime}\right]_{h}}{\Delta \theta_{v}}=\frac{a\left[w^{\prime} \theta_{v}^{\prime}\right]_{s}}{\Delta \theta_{v}}
$$

Equation (18) suggests that the FA moisture, via its effect the $\Delta \theta_{v}$, can modulate the entrainment rate. The value of $\Delta \theta_{v}$ is dependent on $\Delta r$ and $\Delta \theta$ by $\Delta \theta_{v}=\Delta \theta+$ $0.61\left(r_{\mathrm{ML}} \Delta \theta+\theta \Delta r+\Delta \theta \Delta r\right.$ ) (Stull 2012). A drier FA is associated with a more negative $\Delta r$, a smaller $\Delta \theta_{v}$, and stronger entrainment.

The cumulus mass fluxes are parameterized by

$$
M \approx a^{c} w^{*},
$$

where the $a^{c}$ is the cloud fraction of the active growing cloud elements, commonly known as cloud-core area fraction. The $a^{c}$ is parameterized by

$$
a^{c}=0.5+0.36 \arctan \left(1.55 \frac{r_{\mathrm{ML}}-r_{h}^{\mathrm{sat}}}{\sigma_{h}^{r}}\right)
$$

where $r_{h}^{\text {sat }}$ and $\sigma_{h}^{r}$ represent the saturation mixing ratio and the $r$ variance at $h$, respectively. In parentheses $\left(r_{\mathrm{ML}}-r_{h}^{\mathrm{sat}}\right) / \sigma_{h}^{r}$ stands for the moisture deficit normalized by the moisture variance. A zero moisture deficit means a half-cloudy boundary layer $\left(a^{c}=0.5\right)$ in which the cloud-production updrafts are balanced by the cloud-suppression downdrafts for maintaining mass balance. For shallow cumulus, the value of $a^{c}$ is typically several percent (Stevens 2006). The $\sigma_{h}^{r}$ is expressed as

$$
\left(\sigma_{h}^{r}\right)^{2}=-\frac{\overline{\left[w^{\prime} r^{\prime}\right]}}{w^{*}} \frac{\Delta \theta_{v} r}{\Delta \theta_{v} z} h,
$$


TABLE 1. Parameters of the BOMEX case used for solving the ML model.

\begin{tabular}{cccccccc}
\hline \hline$V\left(\mathrm{~m} \mathrm{~s}^{-1}\right)$ & SST $(\mathrm{K})$ & $D\left(\mathrm{~s}^{-1}\right)$ & $r_{800}\left(\mathrm{~g} \mathrm{~kg}^{-1}\right)$ & $\theta_{800}(\mathrm{~K})$ & $p_{s}(\mathrm{hPa})$ & $F_{\text {adv }}^{r}\left(\mathrm{~g} \mathrm{~kg}^{-1} \mathrm{day}^{-1}\right)$ & $F_{\text {adv }}^{\theta}\left(\mathrm{K} \mathrm{day}^{-1}\right)$ \\
\hline 8.75 & 300.4 & $4.3 \times 10^{-6}$ & 4.0 & 308 & 1015 & -1.2 & 0 \\
\hline
\end{tabular}

where $\Delta \theta_{v} z$ is the depth of the transition layer at the $h$ and is assumed as $100 \mathrm{~m}$ for simplicity.

\section{a. Case study}

We start with a case study to gain a preliminary understanding of how the radiative cooling is interacted with other components of the coupled system. The selected case is a shallow cumulus case during BOMEX. The parameters of boundary forcing for this case are shown in Table 1. The values are identical to those used in Neggers et al. (2006) but the major difference is that unlike specifying a constant $Q$ of $2 \mathrm{~K} \mathrm{day}^{-1}$, we relax $Q$, via Eq. (15), as a function of the internal parameters of this coupled model.

Figure 13 shows the temporal evolution of system parameters of the model. An equilibrium state is achieved around 3 days after the initial time. The equilibrium values of the $Q$-sensitive parameters are $\sim 600 \mathrm{~m}$ for $h$ and $\sim 16 \mathrm{~g} \mathrm{~kg}^{-1}$ for $r_{\mathrm{ML}}$, which are in good agreements with the LES results (Siebesma et al. 2003). The equilibrium value of $Q$ is $2.2 \mathrm{~K} \mathrm{day}^{-1}$, which is very close to the $2 \mathrm{~K}$ day $^{-1}$ used in Neggers et al. (2006).

\section{1) Perturbing free-tropospheric moisture}

We perturb $r_{800}$ by $\pm 2 \mathrm{~g} \mathrm{~kg}^{-1}$ at $120 \mathrm{~h}$ when the model has reached an equilibrium state (Fig. 13). The objective is to examine how the $Q$ responds to the FA moisture when the entrainment feedbacks are accounted for. With a drier FA, $Q$ increases immediately because of the greater exposure of ML air to the space. Such a response is transitional. Under a drier FA, the entrainment becomes stronger because of the reduced $\Delta \theta_{v}$. Physically,
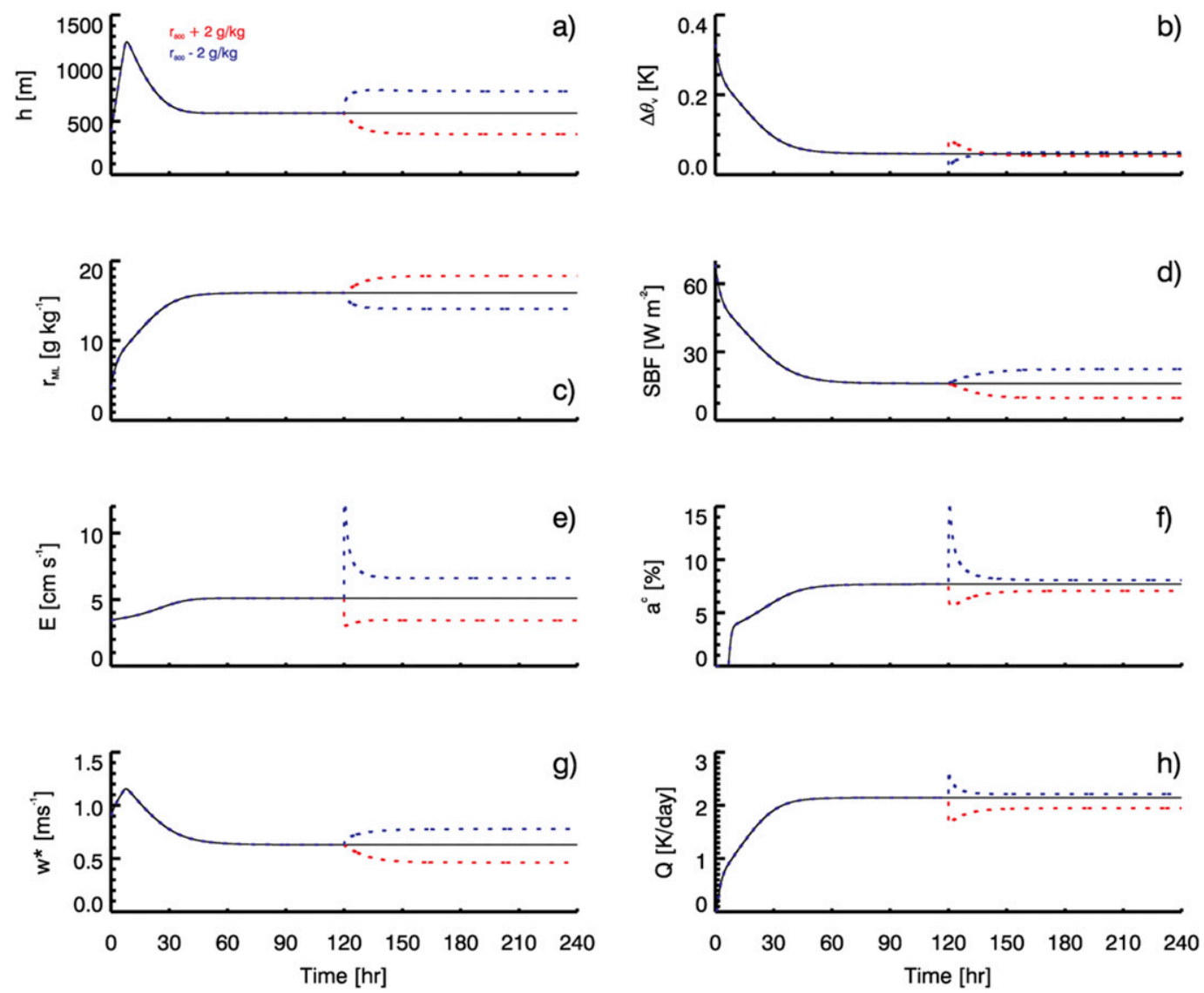

FIG. 13. Temporal variations of various parameters in the simulation of the BOMEX case. The blue and red dashed lines represent the simulated results with $r_{800}$ being perturbed by -2 and $2 \mathrm{~g} \mathrm{~kg}^{-1}$, respectively, at $120 \mathrm{~h}$. 


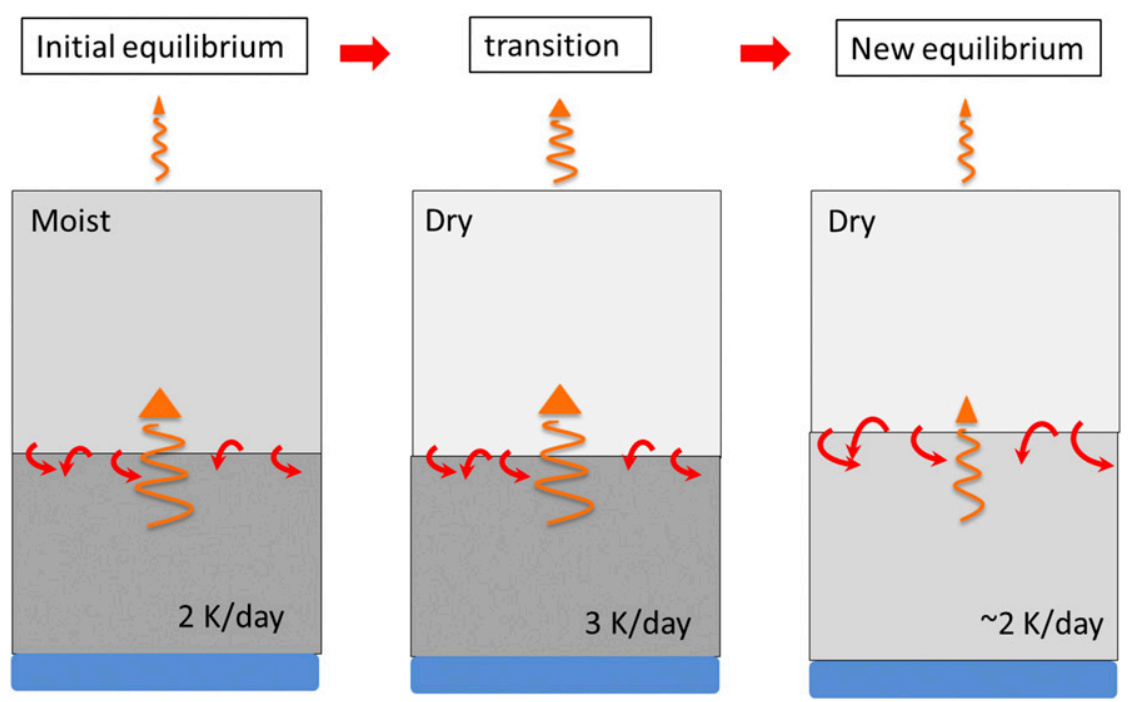

FIG. 14. Cartoon illustrating the resilience of ML radiative cooling to FA moisture. The orange spiral arrows and red arrows represent the radiative fluxes and the entrainment, respectively.

drier air parcels are heavier and thus are more likely to be entrained into the ML. The suddenly enhanced entrainment deepens the ML significantly within a couple of hours. As a negative feedback, when the ML becomes deeper, the absolute temperature at $h$ becomes lower, leading to higher relative humidity at $h$ and higher $a^{c}$ (Ek and Mahrt 1994). Increasing the value of $a^{c}$ enhances $M$ to mitigate the rapid growth of the ML. Meanwhile, the entrainment desiccates the ML. This, on one hand, reduces $E$ by enhancing the buoyancy jump (increase $\Delta \theta_{v}$ ), and, on the other, reduces $M$ by suppressing the cumulus formation (reduce $a^{c}$ ). Finally, the entrainment will balance the combined effect of cumulus mass fluxes and large-scale subsidence, allowing the system to reach a new equilibrium with a drier ML. As was discussed in section 3, a drier ML causes a decrease in $Q$, which offsets the originally increased $Q$. Consequently, $Q$ is close to the original value with only $\sim 0.1 \mathrm{~K} \mathrm{day}^{-1}$ difference. Moistening the FA shows similar processes with an opposite direction.

The mechanism of how $Q$ responds to the perturbation in FA moisture is illustrated in Fig. 14. Desiccating the FA, on one hand, enhances $Q$ via increasing the exposure of ML air to space, and, on the other, weakens $Q$ via enhancing the entrainment drying and decreasing the emitter amount in the ML. At the beginning, the exposure-enhanced radiative effect dominates (middle). Then the ventilation process gradually desiccates the ML, offsetting the initially enhanced $Q$ (right). Eventually, $Q$ responds insensitively to the perturbation of FA moisture. In a nutshell, the entrainment feedback adapts the ML system to maintain a relatively invariant moisture gradient between the ML and the FA, limiting the $Q$ variance.

\section{2) Perturbing SST}

Perturbing the SST by $\pm 1 \mathrm{~K}$ shows that $Q$ is more sensitive to the SST than the FA moisture (Fig. 15). The system responds to the varied SST in a same way as the idealized cases of varying $r_{\mathrm{ML}}$ discussed in section 3 . Increasing the SST enhances the entrainment by increasing the surface buoyancy fluxes and reducing the equilibrium $\Delta \theta_{v}$. This leads to a deeper and drier ML. A drier ML undergoes less radiative cooling because of less emitted amount. However, as the ML deepens, the ML top erodes into a drier atmosphere so that the total moisture burden of the FA decreases, leading to a greater radiative exposure of the ML. This partially offsets the decreasing trend of $Q$. The degree of the offset, however, is not adequate to fully counteract the decrease in $Q$, leaving $Q$ to decrease by $\sim 0.3 \mathrm{~K} \mathrm{day}^{-1}$ with $1-\mathrm{K}$ increase in SST.

\section{b. Composite analysis}

To explore the possible range of the $Q$ for shallowcumulus-topped MLs, we conduct an ensemble of runs by varying SST from 295 to $303 \mathrm{~K}$ and $r_{800}$ from 1 to $7 \mathrm{~g} \mathrm{~kg}^{-1}$. The ML is quite insensitive to large-scale subsidence ( $h$ varies by only tens of meters as a response to a doubling of $D$ ), so we fix it for simplicity. As a reference, we also conduct a companion ensemble of runs in which $Q$ is calculated by full-radiation code. Because running the SBDART model is more computationally expensive than the analytic calculation, $Q$ is updated every $20 \mathrm{~h}$, instead of every time step. 

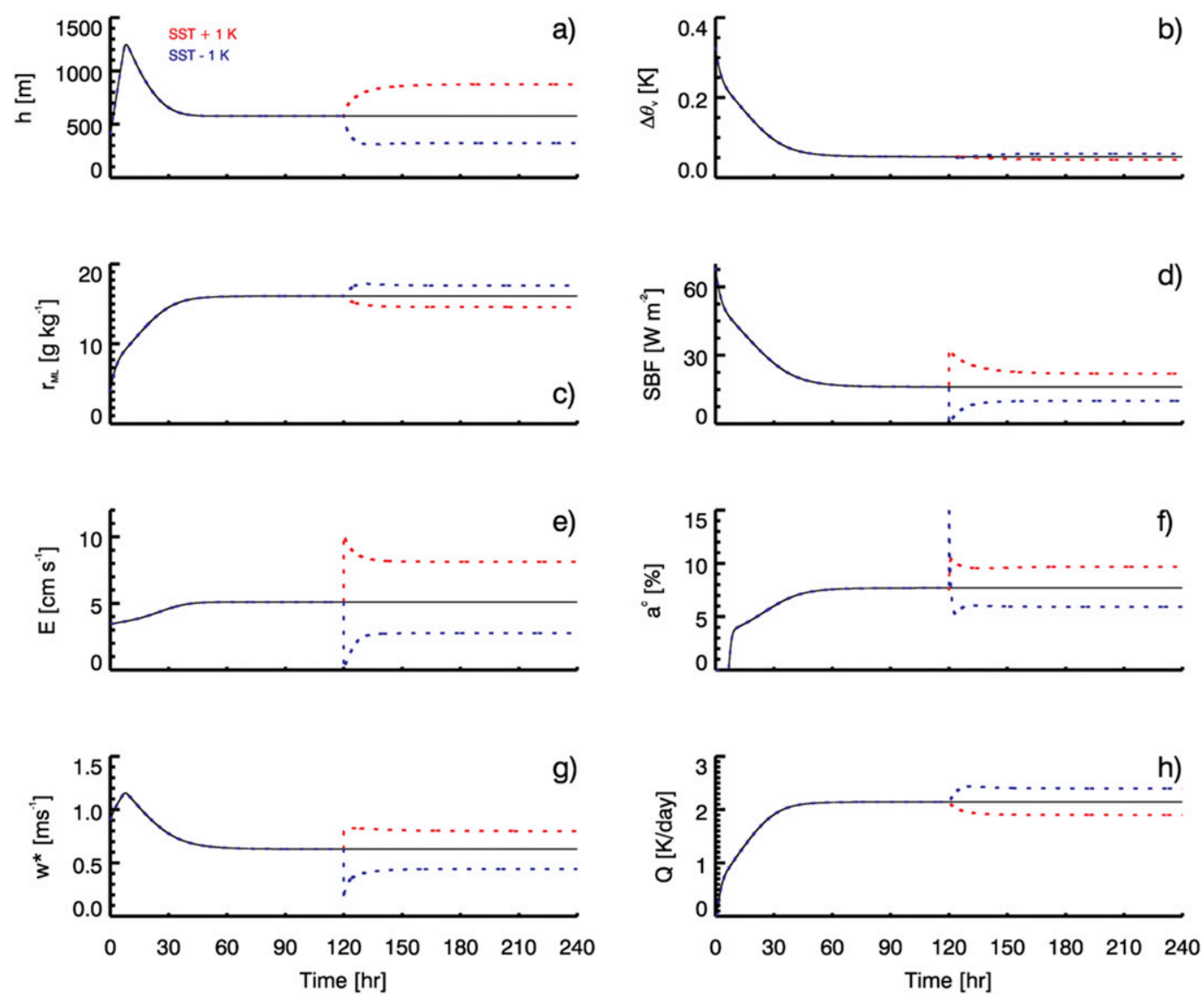

FIG. 15. As in Fig. 13, but the SST is perturbed by -1 and $+1 \mathrm{~K}$.

Figure 16a shows the analytic $Q$ (shading) on a twodimensional parameter space of SST (abscissa) and $r_{800}$ (ordinate). The modeled $h$ (black contours) and $E$ ( $\mathrm{cm} \mathrm{s}^{-1}$, red contours) are superimposed on the plot. The sensitivity of $Q$ to $r_{800}$ experiences a distinct regime shift from a weak-sensitivity regime in highSST condition to strong-sensitivity regime in lowSST condition. The boundary of such a regime shift agrees well with the contours of $E$. As illustrated in Fig. 15, entrainment is the key process that mitigates the radiative impacts of moisture change in FA on $Q$. When the entrainment becomes too weak $\left(<1 \mathrm{~cm} \mathrm{~s}^{-1}\right)$ to effectively mitigate the direct radiative effect, the response of $Q$ to the FA moisture becomes sensitive.

The entrainment rate also regulates how $Q$ responds to SST. As was discussed, decrease in SST strengthens $Q$ via moistening the ML, which is manifest in the large portion of the parameter space in Fig. 16a. Such an effect, however, tends to saturate in weak-entrainment (low SST) situations because of the bounding effect arising from the concave dependence of $E \sim 1 / \Delta \theta_{v}$. As a result, the Planck function starts to dominate the variation of $Q$. Additional reduction in SST reduces the Planck function, weakening $Q$.

The above-described results of $Q$ as a function of SST and FT moisture are consistent with those from simulations in which $Q$ is computed from full-radiation code (Fig. 16b). This further confirms the usage of using Eq. (15) as a simple conceptual framework to understand the radiative cooling process.

Figure 16b shows a range of $Q$ from 1 to $4.5 \mathrm{~K} \mathrm{day}^{-1}$. Not all these values are realistic for shallow-cumulustopped MLs. For example, under a given FA potential temperature, low-SST is associated with a strong temperature inversion that traps a great amount of moisture within the ML. This allows for the formation of layered stratocumulus (Sc) clouds (Wood and Bretherton 2006). The extensive Sc layer will, on one hand, undergo substantially stronger radiative cooling at cloud tops (Wood 2012; Zheng et al. 2016), and, on the other hand, generate stronger turbulent eddies to entrain more FA air to prevent the ML from becoming unrealistically shallow $(<300 \mathrm{~m})$. Neither of the two processes (cloud-generated radiative cooling and turbulence) is accounted for in our ML model. In addition, at the high-SST extreme with a 
a)

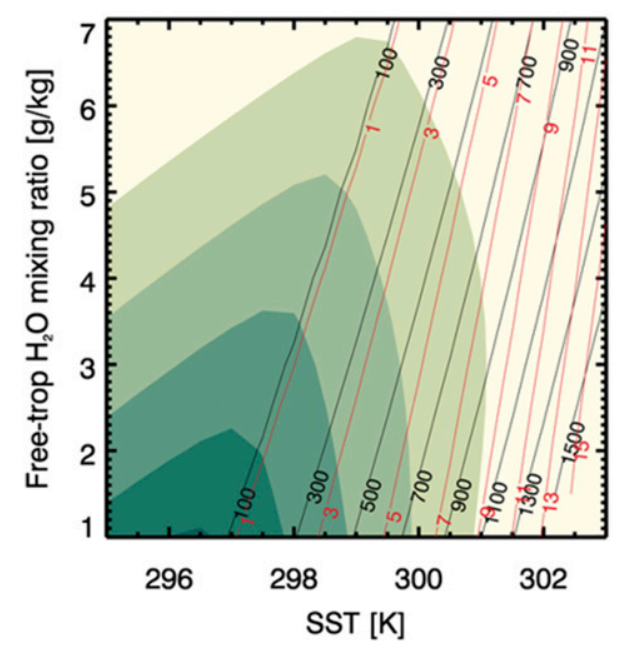

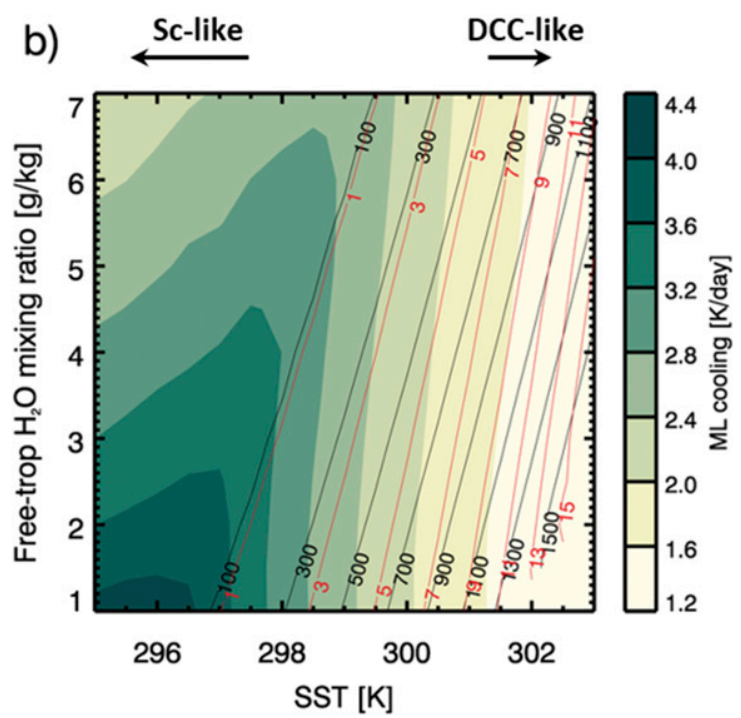

FIG. 16. The radiative cooling rate $Q$ as a function of SST and the FA moisture. The $Q$ is calculated by (a) Eq. (15) and (b) SBDART radiative transfer model. The black and red contours represent the modeled ML height $h(\mathrm{~m})$ and entrainment rate $E\left(\mathrm{~cm} \mathrm{~s}^{-1}\right)$, respectively.

weak temperature inversion, clouds could develop considerably in the vertical to form deep convective clouds (DCCs). The rigorously growing DCCs will extract a considerable amount of air mass out of the subcloud ML to prevent it from growing unrealistically deep $(>1000 \mathrm{~m})$. Apparently, the cloud cover parameterization in the shallow cumulus bulk model [Eq. (20)] is not applicable to deep convection.

The requirement to maintain a shallow cumulus regime further limits the variation of $Q$. Within a reasonable range of $h(300-1000 \mathrm{~m})$ for typical shallow-cumulustopped MLs, $Q=2.01 \pm 0.33 \mathrm{~K} \mathrm{day}^{-1}$, in which the two values represent the average and the standard deviation, respectively. Such a short variation of $Q$ allows the relationship between $w^{*}$ and $z_{b}$ to appear linear according to the discussion in section 2 . This analytically based idea is confirmed by the results from the ML model in Fig. 17 showing the scatterplot of $w^{*}$ versus $h$ for the modeled cases. A strong degree of linearity is manifested for a common range of $h$.

For comparison, the best-fit curve of the relationship between the cloud-base updrafts $w_{b}$ and $z_{b}$ from the observations in Zheng and Rosenfeld (2015) is also superimposed on Fig. 16 (red dashed line). The observations are from the Marine Atmospheric Radiation Measurement (ARM) Global Energy and Water Cycle Experiment-Cloud System Study-Pacific Cross-Section Intercomparison (GPCI) Investigation of Clouds (MAGIC) field campaign. The $z_{b}$ is measured by the ship-based ceilometer. The $w_{b}$ is volume-weighted average of positive vertical velocity measured by W-band
Doppler radar at the cloud bases. To make sense of the comparison, it is necessary to build a bridge between $w_{b}$ and $w^{*}$. A numerical counterpart of the radar-measured $w_{b}$ is the cloud-core updrafts speed, the mean of the pixel-level vertical velocity greater than a certain threshold value (Siebesma et al. 2003; van Stratum et al. 2014). LES simulations show that $w_{b}$ agrees well with

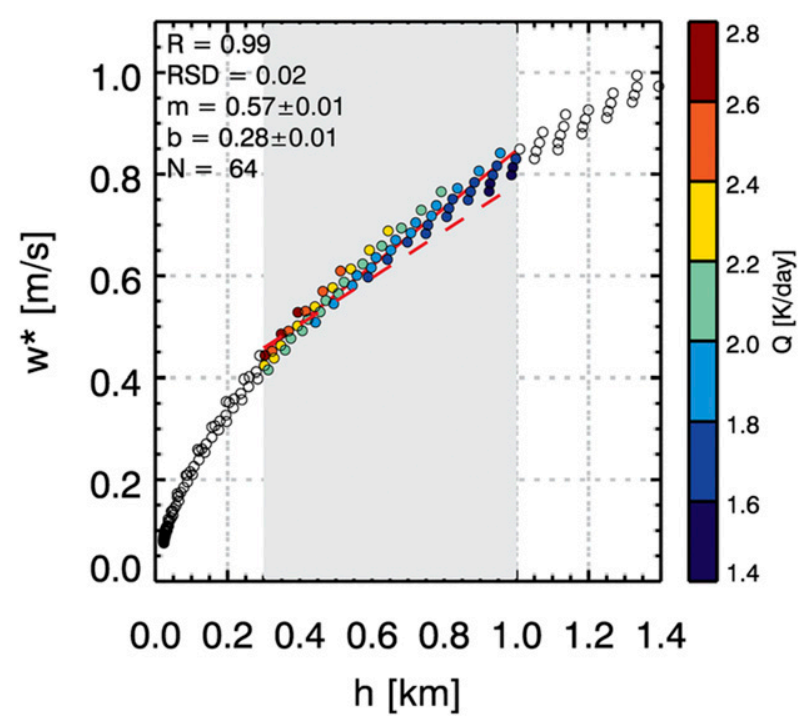

FIG. 17. Plot of $w^{*}$ vs $h$ for the cases simulated by the ML model. RSD stands for the residual standard deviation. The dashed red line marks the best-fit line between the observed relationship between cloud-base updrafts and $z_{b}$ during MAGIC in Zheng and Rosenfeld (2015). 
$w^{*}$ for shallow cumulus (van Stratum et al. 2014). In principle, $w_{b}$ is smaller than $w^{*}$ because when thermals approach the ML top (cloud bases) the updrafts are weakened by the entrainment, viscous drag, and the dilution with the environmental air. In Zheng and Rosenfeld (2015), Doppler radar samples cloudy pixels $100-200 \mathrm{~m}$ above the cloud bases so that the in-cloud latent heating may accelerate the rising parcels. As a result, the values of the observed $w_{b}$ can be comparable to $w^{*}$.

In addition to the absolute value of updrafts, the slope of the relationship is perhaps of more interest because it is conceptually related with $Q$. Based on the results in section 2, a greater $Q$ causes a steeper slope of the $w^{*}-z_{b}$ relation. Since the MAGIC observations show a flatter slope than that from the ML model, it is natural to hypothesize that $Q$ for the MAGIC cases is systematically lower than the BOMEX cases. This hypothesis is physically reasonable for the following two reasons. First, because the MAGIC field campaign was located in the subtropical northeast Pacific where the sea surface is colder than the BOMEX-situated Barbados region. Based on the statistics of the 34 shallow cumulus cases selected during the MAGIC (Zheng and Rosenfeld 2015), the SST varies from 290 to $298 \mathrm{~K}$, which is systematically lower than the modeled BOMEX cases whose SST ranges from 295 to $303 \mathrm{~K}$. For the same relative humidity, the absolute humidity is lower over colder sea surface. With less emitter amount, $Q$ is lower. Second, recalling the section $3 \mathrm{a}$, we specified the moisture profile by assuming a power-law decrease of moisture with altitude for atmosphere above the ML top. In the real world, the existence of a cloud layer at the top of the ML buffers the dramatic decrease of moisture, thus reducing $Q$ via decreasing the radiative exposure. To test this hypothesis, we calculate $Q$ for MAGIC cases using radiosonde observations of the 34 cumulus cases as input for the SBDART radiative transfer model. A lower value of $1.45 \pm 0.22 \mathrm{~K} \mathrm{day}^{-1}$ is obtained, consistent with our hypothesis.

\section{Revisiting the "Bowen ratio argument"}

A question that we can ask is how the above findings relate with WS02's argument that Bowen ratio links the updrafts with $z_{b}$. Up until now, the Bowen ratio has been virtually invariant because the evaporative conductance parameters $\alpha_{\text {LH }}$ have been fixed as 1 [Eq. (17b)]. Although changes in entrainment rate alter the surface thermal and moisture gradients that perturb the instantaneous Bowen ratio, the characteristic property of the sea surface in partitioning surface energy fluxes remains unchanged.
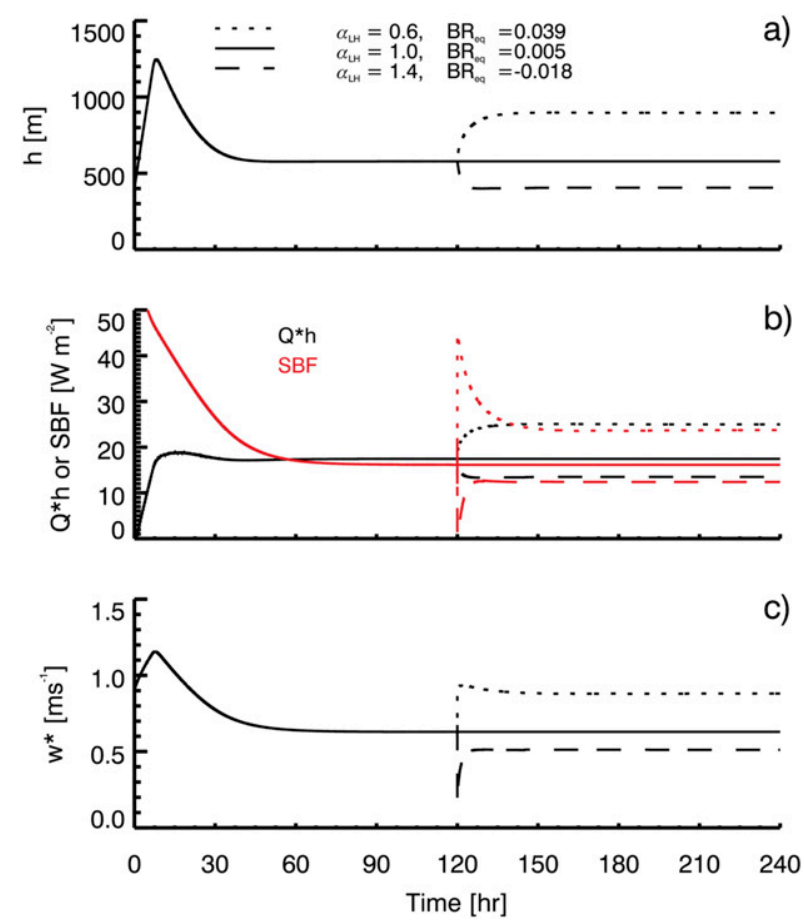

FIG. 18. Temporal evolutions of (a) $h$, (b) $Q h$ and SBF, and (c) $w^{*}$ in the simulation of the BOMEX case with $\alpha_{\mathrm{LH}}$ perturbed by \pm 0.4 at $t=120 \mathrm{~h}$. BR $\mathrm{Bq}_{\mathrm{eq}}$ is the Bowen ratio $\left(F_{\mathrm{SH}} / F_{\mathrm{LH}}\right)$ in the equilibrium at $t=240 \mathrm{~h}$.

To understand this question, I take the BOMEX case and perturb $\alpha_{\mathrm{LH}}$ at $120 \mathrm{~h}$ when the system has reached an equilibrium (Fig. 18). I run nine simulations by perturbing $\alpha_{\mathrm{LH}}$ by $-0.4 \sim 0.4$ with an increment of 0.1 . The SST is adjusted to ensure that the total input of energy from the ocean into the atmosphere $\left(F_{\mathrm{SH}}+F_{\mathrm{LH}}\right)$ remains fixed. Note that the instantaneous Bowen ratio is still free to vary after the perturbation because of the chanes in temperature and moisture gradients near surface, but the equilibrium Bowen ratio $\mathrm{BR}_{\mathrm{eq}}$ ) is controlled by $\alpha_{\mathrm{LH}}$.

Figure 18 shows that the systems reach new equilibria $\sim 1$ day after the perturbations. Initially, a decrease in $\alpha_{\mathrm{LH}}$ (increase in Bowen ratio) causes an immediate increase in $w^{*}$ (short dashed line in Fig. 18c). Given a fixed $F_{\mathrm{SH}}+F_{\mathrm{LH}}$, a higher Bowen ratio leads to stronger $\mathrm{SBF}$ (red in Fig. 18b) and updrafts (Fig. 18c), which deepen the ML (Fig. 18a). This is essentially the argument from WS02. By extracting the $w^{*}$ and $h$ values a half hour after the perturbations $(t=120.5 \mathrm{~h})$ from the nine simulations and comparing them, a positive $w^{*}-h$ correlation with steep slope is noted (blue in Fig. 19). The system at $t=120.5 \mathrm{~h}$, however, is not an energetically balanced system because the rate of enthalpy input into the ML is much larger than the radiative energy loss rate (Fig. 18b). The energy surplus drives the ML to deepen 


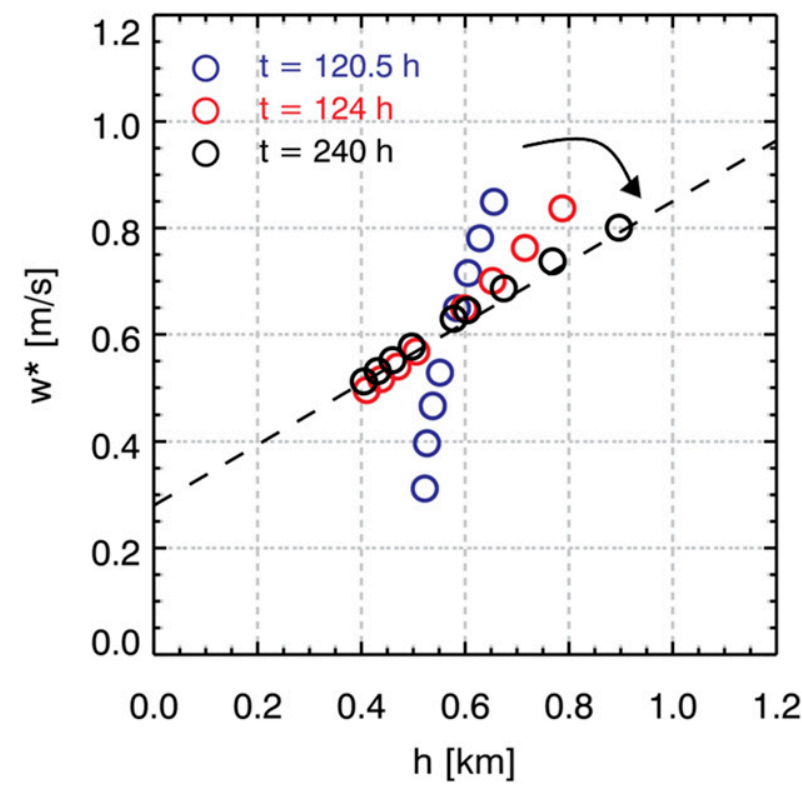

FIG. 19. Plot of $w^{*}$ vs $h$ at $t=120.5$ (blue), 124 (red), and $240 \mathrm{~h}$ (black) for the BOMEX case simulations with $\alpha_{\mathrm{LH}}$ perturbed by -0.4 to +0.4 with an increment of 0.1 at $t=120 \mathrm{~h}$ (nine simulations). The dashed line is the fitted line of $w^{*}$ vs $h$ from Fig. 17.

further. The ML deepening, on one hand, enhances the ML radiative flux divergence and, on the other hand, weakens the initially large surface buoyancy fluxes through the entrainment warming (Fig. 18b). Eventually, the system reaches a new equilibrium with balanced energetics (the SBF is not exactly equivalent to $Q h$ because of the contributions from entrainment and moisture advection terms). In equilibrium $(t=240 \mathrm{~h})$, the slope of the $w^{*}-h$ relationship is markedly flatter than those in transient phases (Fig. 19).

The experiments reveal two prototypes of $w^{*}-h$ relationships: one driven by Bowen ratio (blue in Fig. 19) and one driven by conservation of energetics (black in Fig. 19). The former and latter operate at transient and equilibrium periods, respectively. Initially, the Bowen ratio mechanism (WS02) is responsible for the $w^{*}-h$ relationship. Then, the imbalance between the ML radiative cooling and surface fluxes warming, via entrainment feedback, drives the ML deepening and turbulence weakening of the system to reach a new equilibrium state. In the new equilibrium, the slope of the $w^{*}-h$ relationship (black in Fig. 19) fit well with the $w^{*}-h$ relationship (dashed line) from Fig. 17 in which the Bowen ratio remains virtually unchanged $\left(\alpha_{\mathrm{LH}}\right.$ is fixed as 1$)$. This suggests that the equilibrium $w^{*}-h$ relationship is independent of Bowen ratio. Although in these specific experiments perturbations in $\alpha_{\mathrm{LH}}$ cause variations in $h$ and $w^{*}$, changes in other influential factors such as free-tropospheric moisture and SST can also drive the energy-constrained covariability of $h$ and $w^{*}$ so that the equilibrium $w^{*}-h$ relationship holds (e.g., Fig. 17).

Observations show that the slope of the updrafts $-z_{b}$ relationship is steeper over land than over ocean (Zheng and Rosenfeld 2015). Because a marine boundary layer is closer to equilibrium state than that over land, the theoretical idea presented here is qualitatively consistent with the observations.

\section{Discussion and conclusions}

This study synthesizes radiative transfer and boundary layer theory to establish a theoretical framework for understanding the empirical linear relationship between the convective updrafts and cloud-base height for marine shallow cumulus (Zheng and Rosenfeld 2015). The answers to the three questions posed in the introduction are provided:

- The conservation of energetics for mixed layer (ML) requires that the divergence of radiative fluxes (cooling effect) must balance the surface buoyancy fluxes (warming effect) for maintaining an equilibrium state. As such, given a certain radiative cooling rate per unit mass $Q\left(\mathrm{~K} \mathrm{day}^{-1}\right)$, a deeper ML (higher cloud base) experiences larger radiative flux divergence, which requires stronger surface buoyancy fluxes to balance it, leading to stronger updrafts.

- The response of updrafts to cloud-base height $z_{b}$ manifests linearity. The linearity arises from a limited variation of $Q$ that, as an important regulator of the slope of the updrafts $-z_{b}$ relationship, is resilient to a wide range of changes in the internal and external parameters.

- Because of the resilience of $Q$, the updrafts $-z_{b}$ relationship is insensitive to changes in large-scale environment. The resilience of $Q$ stems from three sources:

1) As the most important absorber in the atmosphere, the water vapor remains strongly absorptive in some wavelengths (e.g., at 6.3 and $>15 \mu \mathrm{m}$ ) even when the water vapor concentration is low. This prevents the vertical gradient of atmospheric transmission, the most important determinant of longwave cooling rate, from becoming too large under dry free atmosphere (Fig. 7).

2) The radiative control of free-tropospheric moisture on $Q$ is largely compensated by its effect on the local entrainment process that regulates the ML moisture budget and the subsequent radiative cooling. Specifically, a drier free atmosphere, on one hand, increases $Q$ by increasing the exposure of the ML air to the cold space, and, on the other hand, decreases $Q$ by entraining drier free-tropospheric air to desiccate the ML. Eventually, $Q$ responds insensitively to the free-tropospheric moisture. 
3) Changes in SST can markedly influence the depth and moisture level of an ML, thus changing $Q$. However, under a certain free-atmosphere potential temperature, changes in SST considerably influence the temperature inversion capping the ML. The shallow cumulus clouds will transition to stratocumulus or deep convective clouds if the temperature inversion becomes too strong or too weak, respectively. Thus, the requirement to maintain the shallow cumulus regime further limits the $Q$ variation.

How the theory relates with the "Bowen ratio argument" by WS02, is discussed. From WS02, under the same radiative forcing of the sea surface, a larger Bowen ratio strengthens the updrafts and deepens the ML. This mechanism, however, does not require the system to be energetically balanced. By increasing the Bowen ratio in an equilibrium cumulus-topped ML model, I found that the WS02's mechanism initially governs the updrafts $-z_{b}$ relationship. However, the imbalance between the ML radiative cooling and the surface warming causes a turbulent energy surplus that drives the system to evolve toward a new equilibrium state in which the updrafts $-z_{b}$ relationship is controlled by the conservation of energy. In a nutshell, the Bowen ratio governs the transient updrafts $-z_{b}$ relationship while the conservation of energetics governs the equilibrium relationship. This finding has significant implications for understanding the relationship over land where the shallow clouds never reach equilibrium states. In that circumstance, the Bowen ratio and the radiative forcing of the surface should play a much more important role. I leave the detailed theoretical analysis of the continental shallow cumulus clouds to Part II of this work.

Acknowledgments. The study was supported by the Department of Energy (DOE) Atmospheric System Research program (DE-SC0018996). The author thanks Zhanqing Li and Daniel Rosenfeld for helpful discussions. I thank Earle Williams and two other anonymous reviewers for their constructive comments that markedly improve the manuscript.

\section{APPENDIX A}

\section{Derivation of $\lambda_{\theta_{v}}$}

With Eqs. (1) and (5), we obtain

$$
\frac{\partial w^{*}}{\partial \theta_{v}}=\frac{1}{3}\left(\frac{g h^{2} Q}{1-a}\right)^{1 / 3} \theta_{v}^{-4 / 3} .
$$

Equations (A1) and (6) allow us to derive the ratio between $\lambda_{\theta_{v}}$ and $\lambda_{h}$ :

$$
\frac{\lambda_{\theta_{v}}}{\lambda_{h}}=\frac{\frac{1}{3}\left(\frac{g h^{2} Q}{1-a}\right)^{1 / 3} \theta_{v}^{-4 / 3} \frac{\partial \theta_{v}}{\partial h}}{\frac{1}{3}\left(\frac{g}{\theta_{v}} \frac{Q}{1-a}\right)^{1 / 3} h^{-1 / 3}}=-\frac{h}{\theta_{v}} \frac{\partial \theta_{v}}{\partial h}=-\frac{\frac{\partial \theta_{v}}{\theta_{v}}}{\frac{\partial h}{h}} .
$$

For tropical and subtropical marine MLs, $h$ ranges from 300 to $1000 \mathrm{~m}$ with mean value of $\sim 600 \mathrm{~m}$. So $\partial h / h \sim 10^{-1}$. The $\partial \theta_{v} / \theta_{v} \sim 10^{-2}$ is typically one magnitude smaller than $\partial h / h$. As a result, $\lambda_{\theta_{\nu}}$ is smaller than $\lambda_{h}$ (and $\lambda_{\mathrm{SBF}}$ ) by one order of magnitude.

\section{APPENDIX B}

\section{Derivation of Eq. (12)}

Based on the derivation of Robinson and Catling (2012), the formulas for $F_{\uparrow}$ and $F_{\downarrow}$ scaled by $\sigma T_{s}^{4}$ at the ML are

$$
\begin{aligned}
& \frac{F_{\uparrow}}{\sigma T_{s}^{4}}=e^{-\left(\tau_{s}-\tau\right)}+\int_{\tau}^{\tau_{s}} d \tau^{\prime} B_{\mathrm{ML}}\left(\tau^{\prime}\right) e^{-\left(\tau^{\prime}-\tau\right)} \\
& \frac{F_{\downarrow}}{\sigma T_{s}^{4}}=\int_{0}^{\tau_{h}} d \tau^{\prime} B_{\mathrm{FA}}\left(\tau^{\prime}\right) e^{-\left(\tau-\tau^{\prime}\right)}+\int_{\tau_{h}}^{\tau} d \tau^{\prime} B_{\mathrm{ML}}\left(\tau^{\prime}\right) e^{-\left(\tau-\tau^{\prime}\right)}
\end{aligned}
$$

At $\tau=\tau_{s}$ and $\tau=\tau_{h}$, substituting Eqs. (B1) into Eq. (10) yields

$$
\begin{aligned}
\frac{\Delta F_{\mathrm{LW}}}{\sigma T_{s}^{4}}= & -\left[1-e^{-\left(\tau_{s}-\tau_{h}\right)}\right] \\
& -\int_{0}^{\tau_{h}} d \tau^{\prime} B_{\mathrm{FA}}\left(\tau^{\prime}\right) e^{-\left(\tau_{h}-\tau^{\prime}\right)}\left[1-e^{-\left(\tau_{s}-\tau_{h}\right)}\right] \\
& +\int_{\tau_{h}}^{\tau_{s}} d \tau^{\prime} B_{\mathrm{ML}}\left(\tau^{\prime}\right) e^{-\left(\tau^{\prime}-\tau_{h}\right)}+\int_{\tau_{h}}^{\tau_{s}} d \tau^{\prime} B_{\mathrm{ML}}\left(\tau^{\prime}\right) e^{-\left(\tau_{s}-\tau^{\prime}\right)}
\end{aligned}
$$

The three terms on the right-hand side of Eq. (B2) stand for the radiative warming from the surface, radiative warming from the FA, and the emission of ML air, respectively. To obtain the "cooling to space" term, we add on the right-hand side two terms that are identical in absolute values but in opposite signs: $\int_{\tau_{h}}^{\tau_{s}} d \tau^{\prime} B_{\mathrm{ML}}\left(\tau^{\prime}\right) e^{-\tau^{\prime}}$ and $-\int_{\tau_{h}}^{\tau_{s}} d \tau^{\prime} B_{\mathrm{ML}}\left(\tau^{\prime}\right) e^{-\tau^{\prime}}$. After some organizations, the latter one will be canceled, yielding the Eq. (12).

\section{REFERENCES}

Albrecht, B. A., A. K. Betts, W. H. Schubert, and S. K. Cox, 1979: Model of the thermodynamic structure of the trade-wind boundary layer: Part I. Theoretical formulation and sensitivity tests. J. Atmos. Sci., 36, 73-89, https://doi.org/10.1175/15200469(1979)036<0073:MOTTSO > 2.0.CO;2. 
Ball, F., 1960: Control of inversion height by surface heating. Quart. J. Roy. Meteor. Soc., 86, 483-494, https://doi.org/10.1002/ qj. 49708637005

Betts, A. K., 1973: Non-precipitating cumulus convection and its parameterization. Quart. J. Roy. Meteor. Soc., 99, 178-196, https://doi.org/10.1002/qj.49709941915.

_ 1976: Modeling subcloud layer structure and interaction with a shallow cumulus layer. J. Atmos. Sci., 33, 2363 2382, https://doi.org/10.1175/1520-0469(1976)033<2363: MSLSAI $>2.0 . \mathrm{CO} ; 2$.

Beucler, T., and T. W. Cronin, 2016: Moisture-radiative cooling instability. J. Adv. Model. Earth Syst., 8, 1620-1640, https:// doi.org/10.1002/2016MS000763.

Bretherton, C. S., 1993: Understanding Albrecht's model of trade cumulus cloud fields. J. Atmos. Sci., 50, 2264-2283, https://doi.org/ 10.1175/1520-0469(1993)050<2264:UAMOTC >2.0.CO;2.

—, and M. C. Wyant, 1997: Moisture transport, lowertropospheric stability, and decoupling of cloud-topped boundary layers. J. Atmos. Sci., 54, 148-167, https://doi.org/ 10.1175/1520-0469(1997)054<0148:MTLTSA>2.0.CO;2.

Cronin, T. W., and M. F. Jansen, 2016: Analytic radiative-advective equilibrium as a model for high-latitude climate. Geophys. Res. Lett., 43, 449-457, https://doi.org/10.1002/2015GL067172.

Deardorff, J. W., 1970: Convective velocity and temperature scales for the unstable planetary boundary layer and for Rayleigh convection. J. Atmos. Sci., 27, 1211-1213, https://doi.org/ 10.1175/1520-0469(1970)027<1211:CVATSF $>2.0 . \mathrm{CO} ; 2$.

Doherty, G. M., and R. E. Newell, 1984: Radiative effects of changing atmospheric water vapour. Tellus, 36B, 149-162, https://doi.org/10.3402/tellusb.v36i3.14885.

Donner, L. J., T. A. O'Brien, D. Rieger, B. Vogel, and W. F. Cooke, 2016: Are atmospheric updrafts a key to unlocking climate forcing and sensitivity? Atmos. Chem. Phys., 16, 12 983-12 992, https://doi.org/10.5194/acp-16-12983-2016.

Ek, M., and L. Mahrt, 1994: Daytime evolution of relative humidity at the boundary layer top. Mon. Wea. Rev., 122, 2709-2721, https:// doi.org/10.1175/1520-0493(1994)122<2709:DEORHA > 2.0.CO;2.

Garratt, J., 1977: Review of drag coefficients over oceans and continents. Mon. Wea. Rev., 105, 915-929, https://doi.org/ 10.1175/1520-0493(1977)105<0915:RODCOO>2.0.CO;2.

Golaz, J.-C., H. Jiang, and W. R. Cotton, 2001: A large-eddy simulation study of cumulus clouds over land and sensitivity to soil moisture. Atmos. Res., 59, 373-392, https://doi.org/10.1016/ S0169-8095(01)00113-2.

Hansen, Z. R., and L. E. Back, 2015: Higher surface Bowen ratios ineffective at increasing updraft intensity. Geophys. Res. Lett., 42, 10 503-10 511, https://doi.org/10.1002/2015GL066878.

Hartmann, D. L., M. E. Ockert-Bell, and M. L. Michelsen, 1992: The effect of cloud type on Earth's energy balance: Global analysis. J. Climate, 5, 1281-1304, https://doi.org/10.1175/15200442(1992)005<1281:TEOCTO > 2.0.CO;2.

Holland, J. Z., and E. M. Rasmusson, 1973: Measurements of the atmospheric mass, energy, and momentum budgets over a 500-kilometer square of tropical ocean. Mon. Wea. Rev., 101, 44-55, https://doi.org/10.1175/1520-0493(1973)101<0044: MOTAME $>2.3 . \mathrm{CO} ; 2$.

Lilly, D. K., 1968: Models of cloud-topped mixed layers under a strong inversion. Quart. J. Roy. Meteor. Soc., 94, 292-309, https://doi.org/10.1002/qj.49709440106.

Mapes, B. E., and P. Zuidema, 1996: Radiative-dynamical consequences of dry tongues in the tropical troposphere. J. Atmos. Sci., 53, 620-638, https://doi.org/10.1175/1520-0469(1996) $053<0620$ :RDCODT $>2.0 . \mathrm{CO} ; 2$.
Naumann, A. K., B. Stevens, C. Hohenegger, and J. P. Mellado, 2017: A conceptual model of a shallow circulation induced by prescribed low-level radiative cooling. J. Atmos. Sci., 74, 31293144, https://doi.org/10.1175/JAS-D-17-0030.1.

Neggers, R., B. Stevens, and J. D. Neelin, 2006: A simple equilibrium model for shallow-cumulus-topped mixed layers. Theor. Comput. Fluid Dyn., 20, 305-322, https://doi.org/10.1007/s00162-006-0030-1.

Nieuwstadt, F. T. M., and R. A. Brost, 1986: The decay of convective turbulence. J. Atmos. Sci., 43, 532-546, https://doi.org/ 10.1175/1520-0469(1986)043<0532:TDOCT > 2.0.CO;2.

Nuijens, L., and B. Stevens, 2012: The influence of wind speed on shallow marine cumulus convection. J. Atmos. Sci., 69, 168184, https://doi.org/10.1175/JAS-D-11-02.1.

Petty, G. W., 2006: A First Course in Atmospheric Radiation. Sundog Publishing, 459 pp.

Rennó, N. O., and Coauthors, 2013: CHASER: An innovative satellite mission concept to measure the effects of aerosols on clouds and climate. Bull. Amer. Meteor. Soc., 94, 685-694, https://doi.org/10.1175/BAMS-D-11-00239.1.

Ricchiazzi, P., S. Yang, C. Gautier, and D. Sowle, 1998: SBDART: A research and teaching software tool for plane-parallel radiative transfer in the Earth's atmosphere. Bull. Amer. Meteor. Soc., 79, 2101-2114, https://doi.org/10.1175/1520-0477(1998)079<2101: SARATS $>2.0 . \mathrm{CO} ; 2$.

Robinson, T. D., and D. C. Catling, 2012: An analytic radiativeconvective model for planetary atmospheres. Astrophys. J., 757, 104, https://doi.org/10.1088/0004-637X/757/1/104.

Romps, D. M., 2017: Exact expression for the lifting condensation level. J. Atmos. Sci., 74, 3891-3900, https://doi.org/10.1175/ JAS-D-17-0102.1.

Rosenfeld, D., 2014: Climate effects of aerosol-cloud interactions. Science, 343, 379-380, https://doi.org/10.1126/science.1247490.

— B. Bischman, Y. Zheng, T. Goren, and D. Giguzin, 2014a: Combined satellite and radar retrievals of drop concentration and CCN at convective cloud base. Geophys. Res. Lett., 41, 3259-3265, https://doi.org/10.1002/2014GL059453.

— precipitation-climate interactions. Rev. Geophys., 52, 750-808, https://doi.org/10.1002/2013RG000441.

_ tion nuclei concentrations by using clouds as CCN chambers. Proc. Natl. Acad. Sci. USA, 113, 5828-5834, https://doi.org/ 10.1073/pnas.1514044113.

_ Y. Yhu, M. Wang, Y. Zheng, T. Goren, and S. Yu, 2019: Aerosol-driven droplet concentrations dominate coverage and water of oceanic low-level clouds. Science, 363, eaav0566, https://doi.org/10.1126/science.aav0566.

Rossow, W. B., and R. A. Schiffer, 1999: Advances in understanding clouds from ISCCP. Bull. Amer. Meteor. Soc., 80, 2261-2288, https://doi.org/10.1175/1520-0477(1999)080<2261: AIUCFI $>2.0 . \mathrm{CO} ; 2$.

Sakradzija, M., and C. Hohenegger, 2017: What determines the distribution of shallow convective mass flux through a cloud base? J. Atmos. Sci., 74, 2615-2632, https://doi.org/10.1175/JASD-16-0326.1.

Schalkwijk, J., H. J. Jonker, and A. P. Siebesma, 2013: Simple solutions to steady-state cumulus regimes in the convective boundary layer. J. Atmos. Sci., 70, 3656-3672, https://doi.org/ 10.1175/JAS-D-12-0312.1.

Seinfeld, J. H., and Coauthors, 2016: Improving our fundamental understanding of the role of aerosol-cloud interactions in the climate system. Proc. Natl. Acad. Sci. USA, 113, 5781-5790, https://doi.org/10.1073/pnas.1514043113. 
Siebesma, A. P., and Coauthors, 2003: A large eddy simulation intercomparison study of shallow cumulus convection. J. Atmos. Sci., 60, 1201-1219, https://doi.org/10.1175/1520-0469(2003) $60<1201$ :ALESIS $>2.0$. CO;2.

Sorbjan, Z., 1997: Decay of convective turbulence revisited. Bound.-Layer Meteor., 82, 503-517, https://doi.org/10.1023/A: 1000231524314.

Stevens, B., 2006: Bulk boundary-layer concepts for simplified models of tropical dynamics. Theor. Comput. Fluid Dyn., 20, 279-304, https://doi.org/10.1007/s00162-006-0032-z.

_ 2007: On the growth of layers of nonprecipitating cumulus convection. J. Atmos. Sci., 64, 2916-2931, https://doi.org/ 10.1175/JAS3983.1.

, H. Brogniez, C. Kiemle, J.-L. Lacour, C. Crevoisier, and J. Kiliani, 2017: Structure and dynamical influence of water vapor in the lower tropical troposphere. Shallow Clouds, Water Vapor, Circulation, and Climate Sensitivity, Springer, 199-225.

Stull, R. B., 2012: An Introduction to Boundary Layer Meteorology. Vol. 13. Springer, $670 \mathrm{pp}$.

Takahashi, K., 2009: Radiative constraints on the hydrological cycle in an idealized radiative-convective equilibrium model. J. Atmos. Sci., 66, 77-91, https://doi.org/10.1175/2008JAS2797.1.

Tennekes, H., and A. G. M. Driedonks, 1981: Basic entrainment equations for the atmospheric boundary layer. Bound.-Layer Meteor., 20, 515-531, https://doi.org/10.1007/BF00122299.

van Driel, R., and H. J. Jonker, 2010: Convective boundary layers driven by nonstationary surface heat fluxes. J. Atmos. Sci., 68 , 727-738, https://doi.org/10.1175/2010JAS3643.1.

van Stratum, B. J., J. Vilá-Guerau de Arellano, C. C. van Heerwaarden, and H. G. Ouwersloot, 2014: Subcloud-layer feedbacks driven by the mass flux of shallow cumulus convection over land. J. Atmos. Sci., 71, 881-895, https://doi.org/ 10.1175/JAS-D-13-0192.1.
Weaver, C., and V. Ramanathan, 1995: Deductions from a simple climate model: Factors governing surface temperature and atmospheric thermal structure. J. Geophys. Res., 100, 11585 11 591, https://doi.org/10.1029/95JD00770.

Williams, E., and S. Stanfill, 2002: The physical origin of the landocean contrast in lightning activity. C. R. Phys., 3, 1277-1292, https://doi.org/10.1016/S1631-0705(02)01407-X.

Wood, R., 2012: Stratocumulus clouds. Mon. Wea. Rev., 140, 2373 2423, https://doi.org/10.1175/MWR-D-11-00121.1.

— stratiform low cloud cover and lower-tropospheric stability. J. Climate, 19, 6425-6432, https://doi.org/10.1175/JCLI3988.1.

Yue, Z., and Coauthors, 2019: Automated Mapping of Convective Clouds (AMCC) thermodynamical, microphysical, and CCN properties from SNPP/VIIRS satellite data. J. Appl. Meteor. Climatol., 58, 887-902, https://doi.org/10.1175/ JAMC-D-18-0144.1.

Zheng, Y., and D. Rosenfeld, 2015: Linear relation between convective cloud base height and updrafts and application to satellite retrievals. Geophys. Res. Lett., 42, 6485-6491, https:// doi.org/10.1002/2015GL064809.

,-- , and Z. Li, 2015: Satellite inference of thermals and cloud-base updraft speeds based on retrieved surface and cloud-base temperatures. J. Atmos. Sci., 72, 2411-2428, https:// doi.org/10.1175/JAS-D-14-0283.1.

,$- \ldots$, and $—$, 2016: Quantifying cloud base updraft speeds of marine stratocumulus from cloud top radiative cooling. Geophys. Res. Lett., 43, 11 407-11413, https://doi.org/10.1002/ 2016 GL071185.

Zhu, Y., D. Rosenfeld, X. Yu, G. Liu, J. Dai, and X. Xu, 2014: Satellite retrieval of convective cloud base temperature based on the NPP/VIIRS imager. Geophys. Res. Lett., 41, 1308-1313, https://doi.org/10.1002/2013GL058970. 